\title{
On the Feynman Path Integral for Nonrelativistic Quantum Electrodynamics
}

\author{
Wataru Ichinose* \\ Department of Mathematical Science, Shinshu University, Matsumoto \\ 390-8621, Japan. \\ E-mail: ichinose@math.shinshu-u.ac.jp
}

The Feynman path integral for regularized nonrelativistic quantum electrodynamics is studied rigorously. We begin with the Lagrangian function of the corresponding classical mechanics and construct the Feynman path integral. In the present paper the electromagnetic potentials are assumed to be periodic with respect to a large box and quantized through their Fourier coefficients with large wave numbers cut off. First, the Feynman path integral with respect to paths on the space of particles and vector potentials is defined rigorously by means of broken line paths under the constraints. Secondly, the Feynman path integral with respect to paths on the space of particles and electromagnetic potentials is also defined rigorously by means of broken line paths and piecewise constant paths without the constraints. This Feynman path integral is stated heuristically in Feynman and Hibbs' book. Thirdly, the vacuum and the state of photons of given momenta and polarizations are expressed concretely as functions of variables consisting of the Fourier coefficients of vector potentials. It is also proved rigorously in terms of distribution theory that the Coulomb potentials between charged particles naturally appear in the above

${ }^{*}$ Research partially supported by Grant-in-Aid for Scientific Research No.16540145 and No.19540175, Ministry of Education, Culture, Sports, Science and Technology, Japanese Government. 
Feynman path integral approach. This shows that the photons give rise to the Coulomb force.

\section{Introduction}

A number of mathematical results on the Feynman path integrals for quantum mechanics have been obtained. On the other hand, the author does not know any mathematical results on the Feynman path integrals for quantum electrodynamics (cf. [2, 23]), written as QED from now on.

The Feynman path integral for the free relativistic scalar boson field was defined rigorously in terms of the infinite dimensional Fresnel integral in [2]. The Chern-Simons functional integral was also defined rigorously, associated with a principal fiber bundle over $R^{3}$ with structure group a compact connected Lie group, as an infinite dimensional distribution in terms of white noise analysis and the applications of its functional integral to the topological quantum field theory were given in [1]. In [27] the interaction of nonrelativistic particles with a scalar boson field was studied. There, the functional integral with respect to paths on the space of particles and the boson field was defined in terms of Markoff processes under the assumption that the mass divided by the imaginary unit and a coupling constant divided by the imaginary unit are positive. As will be seen in the present paper, particles interact with the boson field through the quantized vector potential in QED. On the other hand in [27] particles interact with the boson field through the quantized scalar potential, where the vector potential disappears. This is the most different point between our result and Nelson's one.

The spectra of Hamiltonian operators for nonrelativistic QED models have also been studied (cf. $[12,14,32]$ ). The Hamiltonian operators in these QED models are defined by means of the Coulomb potentials, and creation operators and annihilation operators acting on the bosonic Fock space $\oplus_{n=0}^{\infty} \otimes_{s}^{n}\left(L^{2}\left(R^{3}\right) \oplus\right.$ $L^{2}\left(R^{3}\right)$ ), defined dependently on an infrared and ultraviolet cut-off function in momentum space $R^{3}$, where $L^{2}\left(R^{3}\right)$ is the space of all square integrable functions in $R^{3}$ and $L^{2}\left(R^{3}\right) \oplus L^{2}\left(R^{3}\right)$ expresses the space of all amplitudes 
of momentum of a single photon with polarizations. These QED models are simplified versions of those which are primarily intended in physics (cf. [10, 11, 29, 33]). A functional integral representation for the above nonrelativistic QED model with imaginary time was also obtained by Hiroshima [16] by means of the probabilistic method. We can see from Theorem 3.1 in the present paper that the Hamiltonian operators in $[12,14,16,32]$ are formally like (3.10) in the present paper. But, our presentation (3.10) is exhibited as a partial differential operator. In addition, as will be seen in $\S 5$ of the present paper, creation operators and annihilation operators with given momenta and polarizations acting on $\mathcal{S}^{\prime}\left(R^{4 N}\right)$ are defined and the Hamiltonian operator (3.10) can be written by means of these creation operators and annihilation operators, where $N$ is a positive integer determined from the regularization of QED, $\mathcal{S}\left(R^{4 N}\right)$ denotes the Schwartz space of all rapidly decreasing functions in $R^{4 N}$ and $\mathcal{S}^{\prime}\left(R^{4 N}\right)$ is the dual space of $\mathcal{S}\left(R^{4 N}\right)$. This description of the Hamiltonian operator is the one familiar in the heuristic presentations in physics (cf. [10, 11, 29, 33]).

It is well known that the only translation invariant $\sigma$-additive regular measure on a separable infinite dimensional Banach space is the identically zero measure (cf. Theorem 4 in $\S 5$ of Chapter 4 in [13]). The measure defining heuristically the Feynman path integral is meant to be translation invariant (cf. (7-29) in [11]), so it can not be realized as a $\sigma$-additive regular non trivial measure. As it is known, see, e.g., $[2,15,23]$ the Feynman path integral itself can be realized as a linear functional satisfying certain suitable continuity conditions.

Our aim in the present paper is to define rigorously the Feynman path integral for a regularized nonrelativistic QED (for a physical discussion of QED and its nonrelativistic version, see, e.g., [7, 8, 10, 11, 29]). We begin with the Lagrangian function of the corresponding classical mechanics, differently from the models in $[12,14,16,32]$, and construct rigorously the Feynman path integral. Usually in physics, the Feynman path integral for nonrelativistic QED is only heuristically defined.

In the present paper electromagnetic potentials are assumed to be periodic with respect to a large box in $R^{3}$ and quantized through their Fourier coeffi- 
cients. We note that in the present paper, regrettably, the Fourier coefficients with large wave numbers need to be arbitrarily cut off (ultra-violet cut-off) and we do not take the limit of a box to $R^{3}$. In this double sense our model is regularized.

First, the mathematical definition of the Feynman path integral with respect to paths on the space of particles and vector potentials is given by means of broken line paths under the constraints, i.e. (2.20) in the present paper. These constraints are necessarily introduced in physics (cf. e.g. (9-17) in [11], (A-7) in [29], (13.10) in [32] and (7.38) in [33]) when electrodynamics is quantized from the classical mechanics. It is a reason for introducing the constraints that a momentum canonically conjugate to the scalar potential is absent. See (2.3) in the present paper.

Secondly, without the constraints we give the mathematical definition of the Feynman path integral with respect to paths on the space of particles and electromagnetic potentials by means of broken line paths and piecewise constant paths. This Feynman path integral has been given heuristically by (9-98) in [11]. Our method of defining the Feynman path integral without the constraints is like the one we used before in [20] for defining the phase space Feynman path integral. That is, paths considered on the space of all scalar potentials are determined so that the derivatives of the Lagrangian function with respect to the variables of the scalar potential are piecewise constant (Remark 3.4 in the present paper). The author again emphasize that any definitions of (9-98) in [11] have not been given. So our result may be completely new. We note that our Feynman path integral with respect to paths on the space of particles and electromagnetic potentials can be proved to be equal with the Feynman path integral with respect to paths on the space of particles and vector potentials.

Thirdly, the vacuum and the states of photons with given momenta and polarizations are expressed concretely as functions of variables consisting of the Fourier coefficients of vector potentials. In [11] only the vacuum and the state of a photon with a momentum and a polarization are expressed concretely as functions. Generally, in physics the vacuum and the states of photons with given momenta and polarizations are not considered concretely but rather 
abstractly (cf. [29, 33]). To write down the state of photons concretely, we introduce creation operators and annihilation operators, which can be written concretely as first order partial differential operators, similarly as it is done in white noise analysis in [15]. The results stated above should have many applications, as heuristically suggested in the chapter 9 of [11].

Fourthly, we show in terms of distribution theory that the Coulomb potentials between charged particles appear when the periods of the Fourier series tend to infinity and the cut-off of the Fourier coefficients is removed. This result, which shows that photons yield the Coulomb force, is well known in physics (cf. $[8,11]$ ). In the present paper we give a rigorous proof of this fact in the frame of our model of regularized nonrelativistic QED.

The proof of giving a mathematical definition of the Feynman path integral for nonrelativistic QED with regularization is obtained by means of a somewhat delicate study of oscillatory integral operators, the abstract AscoliArzelà theorem on the weighted Sobolev spaces and the uniqueness to the initial problem for the Schrödinger type equations as in [18, 19, 20, 21].

The proof of expressing the vacuum and the states of photons with given momenta and polarizations concretely is as follows. We first define annihilation operators of photons with given momenta and polarizations by first order differential operators having the Fourier coefficients of vector potentials as variables. Creation operators of photons are defined as the adjoint operators of the annihilation operators. The vacuum is determined from the annihilation operators and the states of photons with given momenta and polarizations are determined from the vacuum by means of the creation operators. For the mathematics related to this see, e.g., [6]. This relies on formal considerations going back to [7].

The proof of the appearance of the Coulomb potentials between charged particles is given by proving the convergence theorem for the Riemann sum of a unbounded function as the discretization parameter in space tends to zero, which will be stated in Proposition 4.3 in the present paper.

Our plan in the present paper is as follows. $§ 2$ is devoted to preliminaries. In $\S 3$ the main results on the Feynman path integral for regularized nonrelativistic QED are stated. In $\S 4$ the appearance of the Coulomb potentials 
between charged particles is proved rigorously in our model. In $\S 5$ the vacuum and the states of photons with given momenta and polarizations are given concretely. $\S 6-\S 9$ are devoted to the proofs of the main results stated in $\S 3$.

\section{Preliminaries}

For a multi-index $\alpha=\left(\alpha_{1}, \ldots, \alpha_{d}\right)$ and $z=\left(z_{1}, \ldots, z_{d}\right) \in R^{d}$ we write $|\alpha|=$ $\sum_{j=1}^{d} \alpha_{j}, z^{\alpha}=z_{1}^{\alpha_{1}} \cdots z_{d}^{\alpha_{d}}, \partial_{z}^{\alpha}=\left(\partial / \partial z_{1}\right)^{\alpha_{1}} \cdots\left(\partial / \partial z_{d}\right)^{\alpha_{d}}$ and $\left\langle z>=\sqrt{1+|z|^{2}}\right.$. Let $L^{2}=L^{2}\left(R^{d}\right)$ be the space of all square integrable functions in $R^{d}$ with inner product $(\cdot, \cdot)$ and norm $\|\cdot\|$.

Let $T>0$ be an arbitrary constant, $t \in[0, T]$ and $x \in R^{3}$. We consider $n$ charged nonrelativistic particles $x^{(j)}(t) \in R^{3}(j=1,2, \ldots, n)$ with mass $m_{j}>0$ and charge $e_{j} \in R$. Let $E(t, x)=\left(E_{1}(t, x), E_{2}(t, x), E_{3}(t, x)\right) \in R^{3}$ be the electric strength and $B(t, x)=\left(B_{1}(t, x), B_{2}(t, x), B_{3}(t, x)\right) \in R^{3}$ the magnetic strength. Then the classical equations of motion of $x^{(j)}(t)$ are given by

$$
\frac{d}{d t} m_{j} \dot{x}^{(j)}(t)=e_{j} E\left(t, x^{(j)}(t)\right)+e_{j} \dot{x}^{(j)}(t) \times B\left(t, x^{(j)}(t)\right), \quad \dot{x}^{(j)}(t)=\frac{d}{d t} x^{(j)}(t) .
$$

Let $\phi(t, x) \in R$ be a scalar potential and $A(t, x) \in R^{3}$ a vector potential. We set

$$
\begin{aligned}
& \vec{x}(t):=\left(x^{(1)}(t), \ldots, x^{(n)}(t)\right) \in R^{3 n} \\
& \dot{\vec{x}}(t):=\left(\dot{x}^{(1)}(t), \ldots, \dot{x}^{(n)}(t)\right) \in R^{3 n}
\end{aligned}
$$

Then the Lagrangian function for particles and the electromagnetic field with the distributional charge density

$$
\rho(t, x)=\sum_{j=1}^{n} e_{j} \delta\left(x-x^{(j)}(t)\right)
$$

and the distributional current density

$$
j(t, x)=\sum_{j=1}^{n} e_{j} \dot{x}^{(j)}(t) \delta\left(x-x^{(j)}(t)\right) \in R^{3}
$$


is given in distributional sense by

$$
\begin{aligned}
\mathcal{L} & \left(t, \vec{x}, \dot{\vec{x}}, A, \dot{A}, \frac{\partial A}{\partial x}, \phi, \frac{\partial \phi}{\partial x}\right) \\
= & \sum_{j=1}^{n} \frac{m_{j}}{2}\left|\dot{x}^{(j)}\right|^{2}-\int \rho(t, x) \phi(t, x) d x+\frac{1}{c} \int j(t, x) \cdot A(t, x) d x \\
& +\frac{1}{8 \pi} \int_{R^{3}}\left(|E(t, x)|^{2}-|B(t, x)|^{2}\right) d x+C \\
= & \sum_{j=1}^{n}\left(\frac{m_{j}}{2}\left|\dot{x}^{(j)}\right|^{2}-e_{j} \phi\left(t, x^{(j)}\right)+\frac{1}{c} e_{j} \dot{x}^{(j)} \cdot A\left(t, x^{(j)}\right)\right) \\
& +\frac{1}{8 \pi} \int_{R^{3}}\left(|E(t, x)|^{2}-|B(t, x)|^{2}\right) d x+C
\end{aligned}
$$

(cf. [11], [32]), where

$$
E=-\frac{1}{c} \frac{\partial A}{\partial t}-\frac{\partial \phi}{\partial x}, \quad B=\nabla \times A,
$$

$\partial \phi / \partial x=\left(\partial \phi / \partial x_{1}, \partial \phi / \partial x_{2}, \partial \phi / \partial x_{3}\right)$ and $C$ is an indefinite constant. It seems that a nontrivial indefinite constant in (2.3) has not been explicitly discussed by anyone before (cf. [11, 29, 32]).

As in $[8,10,29]$ we consider a sufficient large box

$$
V=\left[-\frac{L_{1}}{2}, \frac{L_{1}}{2}\right] \times\left[-\frac{L_{2}}{2}, \frac{L_{2}}{2}\right] \times\left[-\frac{L_{3}}{2}, \frac{L_{3}}{2}\right] \subset R^{3} .
$$

In the present paper as variables we consider all periodic potentials $\phi(t, x)$ and $A(t, x)$ in $x \in R^{3}$ with periods $L_{1}, L_{2}$ and $L_{3}$ satisfying

$$
\nabla \cdot A(t, x)=0 \text { in }[0, T] \times R^{3} \quad \text { (the Coulomb gauge) }
$$

and also

$$
\int_{V} \phi(t, x) d x=0, \quad \int_{V} A(t, x) d x=0 .
$$

Let $|V|=L_{1} L_{2} L_{3}$. We set

$$
k:=\left(\frac{2 \pi}{L_{1}} s_{1}, \frac{2 \pi}{L_{2}} s_{2}, \frac{2 \pi}{L_{3}} s_{3}\right) \quad\left(s_{1}, s_{2}, s_{3}=0, \pm 1, \pm 2, \ldots\right) .
$$


Then, using the Gram and Schmidt method, we can easily determine $\overrightarrow{e_{j}}(k) \in$ $R^{3}(j=1,2)$ such that $\left(\overrightarrow{e_{1}}(k), \overrightarrow{e_{2}}(k), k /|k|\right)$ for all $k \neq 0$ form a set of mutually orthogonal unit vectors in $R^{3}$ and

$$
\overrightarrow{e_{j}}(-k)=-\overrightarrow{e_{j}}(k) \quad(j=1,2)
$$

(cf. p. 448 in [3]). We fix these $\overrightarrow{e_{j}}(k)$ hereafter. Noting (2.5) and (2.6), we can expand $\phi(t, x)$ and $A(t, x)$ formally into the Fourier series

$$
\begin{aligned}
& A\left(x,\left\{a_{1 k}(t)\right\}\right)=\frac{\sqrt{4 \pi}}{|V|} \sum_{k \neq 0}\left\{a_{1 k}(t) \mathrm{e}^{i k \cdot x} \overrightarrow{e_{1}}(k)+a_{2 k}(t) \mathrm{e}^{i k \cdot x} \overrightarrow{e_{2}}(k)\right\}, \\
& \phi\left(x,\left\{\phi_{k}(t)\right\}\right)=\frac{1}{|V|} \sum_{k \neq 0} \phi_{k}(t) \mathrm{e}^{i k \cdot x} .
\end{aligned}
$$

Remark 2.1. Usually in the physical literature (cf. [11, 29]) the condition (2.6) is not stated clearly.

We write

$$
\begin{aligned}
& a_{1 k}=: \frac{a_{1 k}^{(1)}-i a_{1 k}^{(2)}}{\sqrt{2}} \quad(1=1,2), \\
& \phi_{k}=: \phi_{k}^{(1)}-i \phi_{k}^{(2)},
\end{aligned}
$$

where $a_{1 k}^{(\mathrm{i})} \in R$ and $\phi_{k}^{(i)} \in R$, and also the complex conjugate of $a_{\mathrm{l} k}$ as $a_{1 k}^{*}$. Since $A$ and $\phi$ are real valued, the relations

$$
a_{1-k}^{(1)}=-a_{1 k}^{(1)}, \quad a_{1-k}^{(2)}=a_{1 k}^{(2)}, \quad \phi_{-k}^{(1)}=\phi_{k}^{(1)}, \quad \phi_{-k}^{(2)}=-\phi_{k}^{(2)}
$$

hold from (2.8). So, from (2.9) and (2.10) we have

$$
\begin{aligned}
& A\left(x,\left\{a_{1 k}\right\}\right)=\frac{\sqrt{4 \pi}}{|V|} c \sum_{k \neq 0} \sum_{1=1}^{2} \frac{1}{\sqrt{2}}\left(a_{1 k}^{(1)} \cos k \cdot x+a_{1 k}^{(2)} \sin k \cdot x\right) \overrightarrow{e_{1}}(k), \\
& \phi\left(x,\left\{\phi_{k}\right\}\right)=\frac{1}{|V|} \sum_{k \neq 0}\left(\phi_{k}^{(1)} \cos k \cdot x+\phi_{k}^{(2)} \sin k \cdot x\right) .
\end{aligned}
$$

We also write

$$
\begin{aligned}
& \rho_{k}^{(1)}(\vec{x}):=\sum_{j=1}^{n} e_{j} \cos k \cdot x^{(j)}, \\
& \rho_{k}^{(2)}(\vec{x}):=\sum_{j=1}^{n} e_{j} \sin k \cdot x^{(j)} .
\end{aligned}
$$


Determining the constant $C$ in the Lagrangian function (2.3) formally as the infinite constant

$$
\frac{2 \pi}{|V|} \sum_{j=1}^{n} e_{j}^{2} \sum_{k \neq 0} \frac{1}{|k|^{2}}+2 \sum_{k \neq 0} \frac{h c|k|}{2}
$$

we can write $\mathcal{L}$ from (2.3) by means of (2.4), (2.9), (2.10) and (2.15) as

$$
\begin{aligned}
\mathcal{L} & \left(\vec{x}, \dot{\vec{x}},\left\{a_{\mathrm{l} k}\right\},\left\{\dot{a}_{1 k}\right\},\left\{\phi_{k}\right\}\right)=\sum_{j=1}^{n} \frac{m_{j}}{2}\left|\dot{x}^{(j)}\right|^{2} \\
& +\frac{1}{8 \pi|V|} \sum_{k \neq 0}\left\{\sum_{\mathrm{i}=1}^{2}\left(|k|^{2}\left(\phi_{k}^{(\mathrm{i})}\right)^{2}-8 \pi \rho_{k}^{(\mathrm{i})}(\vec{x}) \phi_{k}^{(\mathrm{i})}\right)\right. \\
& \left.+16 \pi^{2} \frac{\sum_{j=1}^{n} e_{j}^{2}}{|k|^{2}}\right\}+\frac{1}{c} \sum_{j=1}^{n} e_{j} \dot{x}^{(j)} \cdot A\left(x^{(j)},\left\{a_{\mathrm{l} k}\right\}\right) \\
& +\frac{1}{2} \sum_{k \neq 0, \mathrm{i}, 1}\left(\frac{\left(\dot{a}_{1 k}^{(\mathrm{i})}\right)^{2}}{2|V|}-\frac{(c|k|)^{2}\left(a_{\mathrm{l} k}^{(\mathrm{i})}\right)^{2}}{2|V|}+\frac{h c|k|}{2}\right) .
\end{aligned}
$$

The reason why we have chosen the indefinite constant $C$ in (2.3) in the way given by (2.18) will be explained in Remark 5.1 .

Remark 2.2. If we do not assume (2.6), we must add $(-1 /|V|)\left(\sum_{j=1}^{n} e_{j}\right) \phi_{0}^{(1)}$ and $\sum_{\mathrm{i}, \mathrm{l}=1,2}\left(\dot{a}_{10}^{(\mathrm{i})}\right)^{2} /(4|V|)$ to $(2.19)$.

If we take into the constraints $\nabla \cdot E=4 \pi \rho$ as in (9-17) in [11] and (7.38) in [33], we have

$$
|k|^{2} \phi_{k}^{(\mathrm{i})}=4 \pi \rho_{k}^{(\mathrm{i})}(\vec{x}) \quad(\mathrm{i}=1,2, k \neq 0)
$$

and $\sum_{j=1}^{n} e_{j}=0$ formally from $(2.1),(2.4)$ and (2.5). But, in the present paper 
we adopt only (2.20) as constraints. Then from (2.16) and (2.17) we have

$$
\begin{aligned}
& \sum_{\mathrm{i}=1}^{2}\left(|k|^{2}\left(\phi_{k}^{(\mathrm{i})}\right)^{2}-8 \pi \rho_{k}^{(\mathrm{i})}(\vec{x}) \phi_{k}^{(\mathrm{i})}\right)+16 \pi^{2} \frac{\sum_{j=1}^{n} e_{j}^{2}}{|k|^{2}} \\
& =-\frac{16 \pi^{2}}{|k|^{2}}\left\{\left(\rho_{k}^{(1)}\right)^{2}+\left(\rho_{k}^{(2)}\right)^{2}-\sum_{j=1}^{n} e_{j}^{2}\right\} \\
& =-\frac{16 \pi^{2}}{|k|^{2}} \sum_{j, l=1, j \neq l}^{n} e_{j} e_{l} \mathrm{e}^{i k \cdot x^{(j)}} \mathrm{e}^{-i k \cdot x^{(l)}} \\
& =-\frac{16 \pi^{2}}{|k|^{2}} \sum_{j, l=1, j \neq l}^{n} e_{j} e_{l} \cos k \cdot\left(x^{(j)}-x^{(l)}\right) .
\end{aligned}
$$

So we get

$$
\begin{aligned}
\mathcal{L}_{c}\left(\vec{x}, \dot{\vec{x}},\left\{a_{\mathrm{l} k}\right\},\left\{\dot{a}_{\mathrm{l} k}\right\}\right)=\sum_{j=1}^{n} \frac{m_{j}}{2}\left|\dot{x}^{(j)}\right|^{2} \\
-\frac{2 \pi}{|V|} \sum_{k \neq 0} \sum_{j, l=1, j \neq l}^{n} \frac{e_{j} e_{l} \cos k \cdot\left(x^{(j)}-x^{(l)}\right)}{|k|^{2}} \\
\quad+\frac{1}{c} \sum_{j=1}^{n} e_{j} \dot{x}^{(j)} \cdot A\left(x^{(j)},\left\{a_{\mathrm{l} k}\right\}\right) \\
\quad+\frac{1}{2} \sum_{k \neq 0, \mathrm{i}, 1}\left(\frac{\left(\dot{a}_{\mathrm{l} k}^{(\mathrm{i})}\right)^{2}}{2|V|}-\frac{(c|k|)^{2}\left(a_{\mathrm{l} k}^{(\mathrm{i})}\right)^{2}}{2|V|}+\frac{h c|k|}{2}\right) .
\end{aligned}
$$

We introduce the weighted Sobolev spaces $B^{a}\left(R^{d}\right):=\left\{f \in L^{2} ;\|f\|_{B^{a}}:=\right.$ $\left.\|f\|+\sum_{|\alpha|=a}\left(\left\|z^{\alpha} f\right\|+\left\|\left(h \partial_{z}\right)^{\alpha} f\right\|\right)<\infty\right\}(a=1,2, \ldots)$. Let $B^{-a}\left(R^{d}\right)$ denote their dual spaces. We set $B^{0}:=L^{2}$. Let $\chi \in C^{\infty}\left(R^{d^{\prime}}\right)$ with compact support such that $\chi(0)=1$. We define the oscillatory integral Os $-\int g\left(\cdot, z^{\prime}\right) d z^{\prime}$ by $\lim _{\epsilon \rightarrow 0} \int \chi\left(\epsilon z^{\prime}\right) g\left(\cdot, z^{\prime}\right) d z^{\prime}$ independently of the choice of $\chi$ pointwise, in the topology of $B^{a}\left(R^{d}\right)$ or in the topology in $\mathcal{S}\left(R^{d}\right)$ (cf. [24]) for a function $g\left(z, z^{\prime}\right)$ in $R^{d} \times R^{d^{\prime}}$, provided the integral involving $\chi$ exists in Lebesgue sense for any $\epsilon>0$. 


\section{Main results}

We arbitrarily cut off the terms of large wave numbers $k$ in (2.22). That is, let $M_{j}(j=1,2,3)$ be arbitrary positive integers such that $M_{2} \leq M_{3}$. We consider

$$
\begin{gathered}
\Lambda_{j}:=\left\{k=\left(\frac{2 \pi}{L_{1}} s_{1}, \frac{2 \pi}{L_{2}} s_{2}, \frac{2 \pi}{L_{3}} s_{3}\right) ; s_{1}^{2}+s_{2}^{2}+s_{3}^{2} \neq 0,\right. \\
\left.\left|s_{1}\right|,\left|s_{2}\right|,\left|s_{3}\right| \leq M_{j}\right\} .
\end{gathered}
$$

Then we can determine $\Lambda_{j}^{\prime}(j=1,2,3)$ such that

$$
\Lambda_{j}=: \Lambda_{j}^{\prime} \cup\left(-\Lambda_{j}^{\prime}\right), \Lambda_{j}^{\prime} \cap\left(-\Lambda_{j}^{\prime}\right)=\text { empty set, } \Lambda_{2}^{\prime} \subseteq \Lambda_{3}^{\prime}
$$

and fix $\Lambda_{j}^{\prime}$ hereafter. Let $N_{j}$ denote the number of elements of the set $\Lambda_{j}^{\prime}$. It follows from (2.13) that $a_{\Lambda_{j}^{\prime}}:=\left\{a_{1 k}^{(\mathrm{i})}\right\}_{k \in \Lambda_{j}^{\prime}, \mathrm{i}, \mathrm{l}} \in R^{4 N_{j}}$ are independent variables (cf. p.154 in [32]).

We also introduce cut-off functions $g(x) \in C^{\infty}\left(R^{3}\right)$ and $\psi \in C^{\infty}(R)$. We consider

$$
\begin{aligned}
& \tilde{\mathcal{L}}_{c}\left(\vec{x}, \dot{\vec{x}},\left\{a_{\mathrm{l} k}\right\},\left\{\dot{a}_{\mathrm{l} k}\right\}\right):=\sum_{j=1}^{n} \frac{m_{j}}{2}\left|\dot{x}^{(j)}\right|^{2} \\
& \quad-\frac{2 \pi}{|V|} \sum_{k \in \Lambda_{1}} \sum_{j, l=1, j \neq l}^{n} \frac{e_{j} e_{l} \cos k \cdot\left(x^{(j)}-x^{(l)}\right)}{|k|^{2}} \\
& \quad+\frac{1}{c} \sum_{j=1}^{n} e_{j} \dot{x}^{(j)} \cdot \tilde{A}\left(x^{(j)}, a_{\Lambda_{2}^{\prime}}\right) \\
& \quad+\frac{1}{2} \sum_{k \in \Lambda_{3}, \mathrm{i}, 1}\left(\frac{\left(\dot{a}_{\mathrm{l} k}^{(\mathrm{i})}\right)^{2}}{2|V|}-\frac{(c|k|)^{2}\left(a_{\mathrm{l} k}^{(\mathrm{i})}\right)^{2}}{2|V|}+\frac{h c|k|}{2}\right)
\end{aligned}
$$

in place of $\mathcal{L}_{c}$ given by (2.22), where $A$ given by (2.14) is replaced with

$$
\begin{aligned}
& \tilde{A}\left(x, a_{\Lambda_{2}^{\prime}}\right)=\frac{\sqrt{4 \pi}}{|V|} c g(x) \sum_{k \in \Lambda_{2}} \sum_{\mathrm{l}=1}^{2} \frac{1}{\sqrt{2}}\left(\psi\left(a_{1 k}^{(1)}\right) \cos k \cdot x\right. \\
& \left.+\psi\left(a_{1 k}^{(2)}\right) \sin k \cdot x\right) \overrightarrow{e_{1}}(k) .
\end{aligned}
$$


We assume $\psi(-\theta)=-\psi(\theta)(\theta \in R)$.

For the sake of simplicity we write $\Lambda^{\prime}:=\Lambda_{3}^{\prime}$ and $N:=N_{3}$. We consider a subdivision

$$
\Delta: 0=\tau_{0}<\tau_{1}<\ldots<\tau_{\nu}=T, \quad|\Delta|:=\max _{1 \leq l \leq \nu}\left(\tau_{l}-\tau_{l-1}\right)
$$

of $[0, T]$. Let $\vec{x} \in R^{3 n}$ and $a_{\Lambda^{\prime}} \in R^{4 N}$ be fixed. We take arbitrarily

$$
\vec{x}^{(0)}, \ldots, \vec{x}^{(\nu-1)} \in R^{3 n}
$$

and

$$
a_{\Lambda^{\prime}}^{(0)}, \ldots, a_{\Lambda^{\prime}}^{(\nu-1)} \in R^{4 N} .
$$

Then, we write the oriented broken line path on $[0, T]$ connecting $\vec{x}^{(l)}$ at $\theta=\tau_{l}\left(l=0,1, \ldots, \nu, \vec{x}^{(\nu)}=\vec{x}\right)$ by $\vec{q}_{\Delta}(\theta) \in R^{3 n}$. Of course, $d \vec{q}_{\Delta}(\theta) / d \theta=$ : $\dot{\vec{q}}_{\Delta}(\theta)$ in distributional sense is in $L^{2}([0, T])$. In the same way we define the broken line path $a_{\Lambda^{\prime} \Delta}(\theta) \in R^{4 N}$ on $[0, T]$ for $a_{\Lambda^{\prime}}^{(0)}, \ldots, a_{\Lambda^{\prime}}^{(\nu-1)}$ and $a_{\Lambda^{\prime}}$. We define $a_{\Lambda \Delta}(\theta) \in R^{8 N}$ by means of (2.13). We write the classical action

$$
\begin{gathered}
S_{c}\left(T, 0 ; \vec{q}_{\Delta}, a_{\Lambda \Delta}\right)=\int_{0}^{T} \tilde{\mathcal{L}}_{c}\left(\vec{q}_{\Delta}(\theta), \dot{\vec{q}}_{\Delta}(\theta),\right. \\
\left.a_{\Lambda \Delta}(\theta), \dot{a}_{\Lambda \Delta}(\theta)\right) d \theta .
\end{gathered}
$$

Let $\rho^{*}>0$ be the constant, which will be defined from $\Lambda_{1}^{\prime}, \Lambda_{2}^{\prime}$ and $\Lambda_{3}^{\prime}$ in Proposition 7.2 of the present paper. See also Remark 7.1. Then we have

THEOREM 3.1. We assume for cut-off functions $g(x)$ and $\psi(\theta)$ in (3.4) that for any $l=1,2, \ldots$ and any multi-index $\alpha$ there exist constants $\delta_{l}>0$ and $\delta_{\alpha}>0$ satisfying

$$
\left|\partial_{\theta}^{l} \psi(\theta)\right| \leq C_{l}<\theta>^{-\left(1+\delta_{l}\right)}, \theta \in R
$$

and

$$
\left|\partial_{x}^{\alpha} g(x)\right| \leq C_{\alpha}<x>^{-\left(1+\delta_{\alpha}\right)}, x \in R^{3} .
$$


Let $|\Delta| \leq \rho^{*}$ and $f\left(\vec{x}, a_{\Lambda^{\prime}}\right) \in B^{a}\left(R^{3 n+4 N}\right)(a=0,1,2, \ldots)$. Then,

$$
\begin{aligned}
& \left\{\prod_{l=1}^{\nu}\left(\prod_{j=1}^{n}{\sqrt{\frac{m_{j}}{2 \pi i \hbar\left(\tau_{l}-\tau_{l-1}\right)}}}^{3}\right){\sqrt{\frac{1}{2 \pi i|V| h\left(\tau_{l}-\tau_{l-1}\right)}}}^{4 N}\right\} \\
& \times O s-\int \cdots \int\left(\exp i h^{-1} S_{c}\left(T, 0 ; \vec{q}_{\Delta}, a_{\Lambda \Delta}\right)\right) f\left(\vec{q}_{\Delta}(0),\right. \\
& \left.a_{\Lambda^{\prime} \Delta}(0)\right) d \vec{x}^{(0)} \cdots d \vec{x}^{(\nu-1)} d a_{\Lambda^{\prime}}^{(0)} \cdots d a_{\Lambda^{\prime}}^{(\nu-1)}
\end{aligned}
$$

is well defined in $B^{a}\left(R^{3 n+4 N}\right)$, which we write as $\left(C_{\Delta}(T, 0) f\right)\left(\vec{x}, a_{\Lambda^{\prime}}\right)$ or $\iint\left(\exp i \hbar^{-1} S_{c}\left(T, 0 ; \vec{q}_{\Delta}, a_{\Lambda \Delta}\right)\right) f\left(\vec{q}_{\Delta}(0), a_{\Lambda^{\prime} \Delta}(0)\right) \mathcal{D} \vec{q}_{\Delta} \mathcal{D} a_{\Lambda^{\prime} \Delta} . \quad$ In addition, as $|\Delta|$ tends to 0 , the function $\left(\mathcal{C}_{\Delta}(T, 0) f\right)\left(\vec{x}, a_{\Lambda^{\prime}}\right)$ converges to a limit which we call the Feynman path integral $\iint\left(\exp i h^{-1} S_{c}\left(T, 0 ; \vec{q}, a_{\Lambda}\right)\right) f\left(\vec{q}(0), a_{\Lambda^{\prime}}(0)\right)$ $\mathcal{D} \vec{q} \mathcal{D} a_{\Lambda^{\prime}}$ in $B^{a}\left(R^{3 n+4 N}\right)$. We can also see that this limit is $B^{a}$-valued continuous and $B^{a-2}$-valued continuously differentiable in $T \in(0, \infty)$, and satisfies the Schrödinger type equation

$$
i \hbar \frac{\partial}{\partial t} u(t)=H(t) u(t)
$$

with $u(0)=f$, where

$$
\begin{gathered}
H(t)=\sum_{j=1}^{n} \frac{1}{2 m_{j}}\left|\frac{h}{i} \frac{\partial}{\partial x^{(j)}}-\frac{e_{j}}{c} \tilde{A}\left(x^{(j)}, a_{\Lambda_{2}^{\prime}}\right)\right|^{2} \\
+\frac{2 \pi}{|V|} \sum_{k \in \Lambda_{1}} \sum_{j, l=1, j \neq l}^{n} \frac{e_{j} e_{l} \cos k \cdot\left(x^{(j)}-x^{(l)}\right)}{|k|^{2}} \\
+\sum_{k \in \Lambda^{\prime}, \mathrm{i}, 1}\left\{\frac{|V|}{2}\left(\frac{h}{i} \frac{\partial}{\partial a_{1 k}^{(\mathrm{i})}}\right)^{2}+\frac{(c|k|)^{2}}{2|V|}\left(a_{1 k}^{(\mathrm{i})}\right)^{2}-\frac{h c|k|}{2}\right\} .
\end{gathered}
$$

Remark 3.1. Let us determine the indefinite constant $C$ in (2.3) by

$$
\frac{2 \pi}{|V|} \sum_{j=1}^{n} e_{j}^{2} \sum_{k \in \Lambda_{1}} \frac{1}{|k|^{2}}+2 \sum_{k \in \Lambda_{3}} \frac{h c|k|}{2}
$$

and cut off the terms of large wave numbers $k$ in (2.19) by introducing $\Lambda_{j}(j=$ $1,2,3)$. Then we get $(3.3)$ again, taking into the account the constraints $(2.20)$. 
Remark 3.2. Let $0<\epsilon \leq 1$ and $g_{\epsilon}(x) \in C^{\infty}\left(R^{3}\right)$ satisfy (3.7) for all $\alpha$. Let $U_{\epsilon}(t, 0) f(0 \leq t \leq T)$ denote the Feynman path integral defined in Theorem 3.1 for $f \in B^{a}\left(R^{3 n+4 N}\right)$. Suppose that $\partial_{x}^{\alpha} g_{\epsilon}(x)$ are uniformly bounded with respect to $0<\epsilon \leq 1$ in $R^{3 n}$ for all $\alpha$ and that $\partial_{x}^{\alpha} g_{\epsilon}(x)$ converges to $\partial_{x}^{\alpha} 1$ pointwise in $R^{3 n}$ for all $\alpha$ as $\epsilon$ tends to zero. Then we can prove that as $\epsilon$ tends to zero, $U_{\epsilon}(t, 0) f$ converges to the solution of $(3.9)$ with $u(0)=f$, where $g(x)$ in (3.4) is replaced with 1 , in $B^{a}$ uniformly in $t \in[0, T]$. In this way we can remove the cut-off function $g(x)$ in (3.4). This result will be published in $[22]$.

Remark 3.3. Let $0 \leq t_{0} \leq t \leq T$. For $f \in B^{a}\left(R^{3 n+4 N}\right)(a=0,1,2, \ldots)$ we define $\mathcal{C}_{\Delta}\left(t, t_{0}\right) f$ with $\mathcal{C}_{\Delta}\left(t_{0}, t_{0}\right) f=f$ as in (3.8). See (9.3) in the present paper for the precise definition. As will be seen from the proof of Theorem 3.1 of the present paper, under the assumptions of Theorem $3.1\left(\mathcal{C}_{\Delta}\left(t, t_{0}\right) f\right)\left(\vec{x}, a_{\Lambda^{\prime}}\right)$ is well defined in $B^{a}$ and $\lim _{|\Delta| \rightarrow 0} \mathcal{C}_{\Delta}\left(t, t_{0}\right) f$ exists in $B^{a}$ uniformly in $0 \leq t_{0} \leq$ $t \leq T$, which satisfies the Schödinger type equation (3.9) with $u\left(t_{0}\right)=f$.

In place of $\mathcal{L}$ expressed by (2.19) we consider

$$
\begin{aligned}
\tilde{\mathcal{L}} & \left(\vec{x}, \dot{\vec{x}},\left\{a_{1 k}\right\},\left\{\dot{a}_{1 k}\right\},\left\{\phi_{k}\right\}\right):=\sum_{j=1}^{n} \frac{m_{j}}{2}\left|\dot{x}^{(j)}\right|^{2} \\
& +\frac{1}{8 \pi|V|} \sum_{k \in \Lambda_{1}}\left\{\sum_{\mathrm{i}=1}^{2}\left(|k|^{2}\left(\phi_{k}^{(\mathrm{i})}\right)^{2}-8 \pi \rho_{k}^{(\mathrm{i})}(\vec{x}) \phi_{k}^{(\mathrm{i})}\right)\right. \\
& \left.+16 \pi^{2} \frac{\sum_{j=1}^{n} e_{j}^{2}}{|k|^{2}}\right\}+\frac{1}{c} \sum_{j=1}^{n} e_{j} \dot{x}^{(j)} \cdot \tilde{A}\left(x^{(j)}, a_{\Lambda_{2}^{\prime}}\right) \\
& +\frac{1}{2} \sum_{k \in \Lambda_{3}, \mathrm{i}, 1}\left(\frac{\left(\dot{a}_{1 k}^{(\mathrm{i})}\right)^{2}}{2|V|}-\frac{(c|k|)^{2}\left(a_{1 k}^{(\mathrm{i})}\right)^{2}}{2|V|}+\frac{h c|k|}{2}\right)
\end{aligned}
$$

by means of $(3.4)$ as in $\tilde{\mathcal{L}}_{c}$.

Let $\vec{q}_{\Delta}(\theta) \in R^{3 n}, a_{\Lambda^{\prime} \Delta}(\theta) \in R^{4 N}$ and $a_{\Lambda \Delta}(\theta) \in R^{8 N}$ be the broken line paths defined before. Let $\vec{\xi}_{k}:=\left(\xi_{k}^{(1)}, \xi_{k}^{(2)}\right) \in R^{2}$ for $k \in \Lambda_{1}^{\prime}$. Take $\vec{\xi}_{k}^{(0)}, \vec{\xi}_{k}^{(1)}, \ldots$ and $\vec{\xi}_{k}^{(\nu-1)}$ in $R^{2}$ arbitrarily. Set $\rho_{k}(\vec{x}):=\left(\rho_{k}^{(1)}(\vec{x}), \rho_{k}^{(2)}(\vec{x})\right)$ 
by means of (2.16) and (2.17). Then, we define the path

$$
\phi_{k \Delta}(\theta):=\vec{\xi}_{k}^{(l)}+\frac{4 \pi \rho_{k}\left(\vec{q}_{\Delta}(\theta)\right)}{|k|^{2}} \in R^{2}, \tau_{l-1}<\theta \leq \tau_{l}
$$

$(l=1,2, \ldots, \nu)$, where $\phi_{k \Delta}(0):=\lim _{\theta \rightarrow 0+0} \phi_{k \Delta}(\theta)$. We set $\phi_{\Lambda_{1}^{\prime} \Delta}(\theta):=\left\{\phi_{k \Delta}(\theta)\right\}_{k \in \Lambda_{1}^{\prime}}$ $\in R^{2 N_{1}}$. We define $\phi_{\Lambda_{1} \Delta}(\theta) \in R^{4 N_{1}}$ by means of $(2.13)$. Let $S\left(T, 0 ; \vec{q} \Delta, a_{\Lambda \Delta}, \phi_{\Lambda_{1} \Delta}\right)$ be the classical action for $\tilde{\mathcal{L}}\left(\vec{x}, \dot{\vec{x}},\left\{a_{\mathrm{l} k}\right\},\left\{\dot{a}_{\mathrm{lk}}\right\},\left\{\phi_{k}\right\}\right)$ given by (3.11).

TheOrem 3.2. Let $|\Delta| \leq \rho^{*}$ and $f\left(\vec{x}, a_{\Lambda^{\prime}}\right) \in B^{a}\left(R^{3 n+4 N}\right)(a=0,1,2, \ldots)$. Then, under the assumptions of Theorem 3.1 the function

$$
\begin{aligned}
& {\left[\prod _ { l = 1 } ^ { \nu } \left\{\left(\prod_{j=1}^{n}{\sqrt{\frac{m_{j}}{2 \pi i \hbar\left(\tau_{l}-\tau_{l-1}\right)}}}^{3}\right){\sqrt{\frac{1}{2 \pi i h|V|\left(\tau_{l}-\tau_{l-1}\right)}}}^{4 N}\right.\right.} \\
& \left.\left.\times \prod_{k \in \Lambda_{1}^{\prime}} \frac{|k|^{2}\left(\tau_{l}-\tau_{l-1}\right)}{4 i \pi^{2} h|V|}\right\}\right] O s-\int \cdots \int\left(\exp i h^{-1} S(T, 0 ;\right. \\
& \left.\left.\vec{q}_{\Delta}, a_{\Lambda \Delta}, \phi_{\Lambda_{1} \Delta}\right)\right) f\left(\vec{q}_{\Delta}(0), a_{\Lambda^{\prime} \Delta}(0)\right) d \vec{x}^{(0)} \cdots d \vec{x}^{(\nu-1)} \\
& \cdot d a_{\Lambda^{\prime}}^{(0)} \cdots d a_{\Lambda^{\prime}}^{(\nu-1)} \prod_{k \in \Lambda_{1}^{\prime}} d \vec{\xi}_{k}^{(0)} d \vec{\xi}_{k}^{(1)} \cdots d \vec{\xi}_{k}^{(\nu-1)}
\end{aligned}
$$

is well defined in $B^{a}\left(R^{3 n+4 N}\right)$ and is equal to

$$
\begin{aligned}
& \iint\left(\exp i h^{-1} S_{c}\left(T, 0 ; \vec{q}_{\Delta}, a_{\Lambda \Delta}\right)\right) \\
& \quad \times f\left(\vec{q}_{\Delta}(0), a_{\Lambda^{\prime} \Delta}(0)\right) \mathcal{D} \vec{q} \Delta \mathcal{D} a_{\Lambda^{\prime} \Delta}
\end{aligned}
$$

defined by (3.8) in Theorem 3.1. So it follows from Theorem 3.1 that as $|\Delta| \rightarrow$ 0, then (3.13) converges to the Feynman path integral

$$
\iiint\left(\exp i h^{-1} S\left(T, 0 ; \vec{q}, a_{\Lambda}, \phi_{\Lambda_{1}}\right)\right) f\left(\vec{q}(0), a_{\Lambda^{\prime}}(0)\right) \mathcal{D} \vec{q} \mathcal{D} a_{\Lambda^{\prime}} \mathcal{D} \phi_{\Lambda_{1}^{\prime}}
$$

in $B^{a}\left(R^{3 n+4 N}\right)$, which satisfies the Schrödinger type equation (3.9) with $u(0)=$ $f$.

The Feynman path integral (3.14) is given heuristically in $§ 9-8$ in FeynmanHibbs [11]. 
Remark 3.4. As was noted in the introduction, the constraints (2.20) are not needed in Theorem 3.2 above. The path $\phi_{k \Delta}(\theta)$ defined by (3.12) is determined so that $\partial \tilde{\mathcal{L}}\left(\vec{q}_{\Delta}(\theta), \dot{\vec{q}}_{\Delta}(\theta), a_{\Lambda \Delta}(\theta), \dot{a}_{\Lambda \Delta}(\theta), \phi_{\Lambda_{1} \Delta}(\theta)\right) / \partial \phi_{k}^{(\mathrm{i})}(\mathrm{i}=1,2)$ are piecewise constant.

Remark 3.5. We take $f \in \mathcal{S}\left(R^{3 n+4 N}\right)$ and set $M_{0}=[(3 n+4 N) / 2]+1$, where [.] denotes Gauss' symbol. Let $\zeta=(x, X)$, and $\alpha$ and $\beta$ multi-indices. Then, the Sobolev inequality shows

$$
\sup _{\zeta \in R^{3 n+4 N}}\left|\zeta^{\alpha} \partial_{\zeta}^{\beta} f(\zeta)\right| \leq\left\|\zeta^{\alpha} \partial_{\zeta}^{\beta} f\right\|+\sum_{|\kappa|=M_{0}}\left\|\partial_{\zeta}^{\kappa}\left(\zeta^{\alpha} \partial_{\zeta}^{\beta} f\right)\right\| .
$$

It follows from Lemma 2.4 with $a=b=1$ in [17] or as in the proof of (7.14) in the present paper that the rhs of the above is bounded by $C_{\alpha, \beta}\|f\|_{B^{|\alpha+\beta|+M_{0}}}$ with a constant $C_{\alpha, \beta}$. Hence, for $|\Delta| \leq \rho^{*}$ the functions (3.8), (3.13), the limit of (3.8) as $|\Delta| \rightarrow 0$ and the limit of (3.13) as $|\Delta| \rightarrow 0$ are well defined in $\mathcal{S}$, so pointwise.

Remark 3.6. We write (3.13) as $G_{\Delta}(T, 0) f$. Let $0 \leq t_{0} \leq t \leq T$. For $f \in B^{a}\left(R^{3 n+4 N}\right)(a=0,1,2, \ldots)$ we can define $G_{\Delta}\left(t, t_{0}\right) f$ as in (3.13) in the same way that $\mathcal{C}_{\Delta}\left(t, t_{0}\right) f$ is defined in Remark 3.3. See also (9.20) in the present paper for the precise definition. As will be seen in the proof of Theorem 3.2, under the assumptions of Theorem $3.1 G_{\Delta}\left(t, t_{0}\right) f$ is well defined in $B^{a}$ and is equal to $\mathcal{C}_{\Delta}\left(t, t_{0}\right) f$.

We consider an external electromagnetic field $E_{\mathrm{ex}}(t, x)=\left(E_{\mathrm{ex} 1}(t, x), E_{\mathrm{ex} 2}(t, x)\right.$, $\left.E_{\mathrm{ex} 3}(t, x)\right) \in R^{3}$ and $B_{\mathrm{ex}}(t, x)=\left(B_{\mathrm{ex} 1}(t, x), B_{\mathrm{ex} 2}(t, x), B_{\mathrm{ex} 3}(t, x)\right) \in R^{3}$ such that $\partial_{x}^{\alpha} E_{\operatorname{exj}}(t, x), \partial_{x}^{\alpha} B_{\operatorname{exj}}(t, x)$ and $\partial_{t} B_{\operatorname{exj}}(t, x)(j=1,2,3)$ are continuous in $[0, T] \times R^{n}$ for all $\alpha$. Let $\phi_{\text {ex }}(t, x) \in R$ and $A_{\text {ex }}(t, x) \in R^{3}$ be the electromagnetic potential to $E_{\text {ex }}$ and $B_{\text {ex }}$. Then we obtain Theorem 3.3 below. Though Theorem 3.3 gives the generalization of Theorems 3.1 and 3.2, the results are stated separately from Theorems 3.1 and 3.2 to avoid confusion.

We replace $\tilde{A}\left(x^{(j)}, a_{\Lambda_{2}^{\prime}}\right)$ in (3.3), (3.10) and (3.11) by $\tilde{A}\left(x^{(j)}, a_{\Lambda_{2}^{\prime}}\right)+A_{\mathrm{ex}}\left(t, x^{(j)}\right)$. Moreover we add $-\sum_{j=1}^{n} e_{j} \phi_{\mathrm{ex}}\left(t, x^{(j)}\right)$ to $(3.3)$ and (3.11), and $\sum_{j=1}^{n} e_{j} \phi_{\mathrm{ex}}\left(t, x^{(j)}\right)$ to (3.10), respectively. Then we have

TheOrem 3.3. Besides the assumptions of Theorem 3.1 we suppose as in Ichinose [19, 20, 21] that for any $\alpha \neq 0$ there exist constants $C_{\alpha}$ and $\delta_{\alpha}>0$ 
satisfying

$$
\left|\partial_{x}^{\alpha} E_{e x j}(t, x)\right| \leq C_{\alpha},\left|\partial_{x}^{\alpha} B_{e x j}(t, x)\right| \leq C_{\alpha}<x>^{-\left(1+\delta_{\alpha}\right)}
$$

and

$$
\left|\partial_{x}^{\alpha} A_{e x j}(t, x)\right| \leq C_{\alpha},\left|\partial_{x}^{\alpha} \phi_{e x}(t, x)\right| \leq C_{\alpha}<x>
$$

for $j=1$, 2 and 3 in $[0, T] \times R^{n}$. Then, the same assertions as in Theorems 3.1 and 3.2 hold.

Remark 3.7. It follows from Lemma 6.1 in [19] that under the assumptions (3.15) there exist $A_{\text {ex }}$ and $\phi_{\text {ex }}$ satisfying (3.16).

\section{The appearance of the Coulomb potentials}

We will show rigorously that the Coulomb potentials appear as the limit of the second term on the rhs of (3.3) and the limit of the second term on the rhs of (3.10). This result is well known as a heuristic result in physics (cf. [8, 11]). We will give a rigorous proof in our model. In the Hamiltonian operators of QED models in $[12,14,16,32]$ the Coulomb potentials are assumed from the beginning. Our proof is somewhat delicate.

TheOrem 4.1. Let $L_{j}(j=1,2,3)$ tend to $\infty$ under the condition

$$
\frac{1}{m_{0}} \leq \frac{L_{i}}{L_{j}} \leq m_{0}, \quad i, j=1,2,3
$$

for a constant $m_{0} \geq 1$. Then we have

$$
\begin{aligned}
& \lim _{L_{1}, L_{2}, L_{3} \rightarrow \infty} \lim _{M_{1} \rightarrow \infty} \frac{2 \pi}{|V|} \sum_{k \in \Lambda_{1}} \sum_{j, l=1, j \neq l}^{n} \frac{e_{j} e_{l} \cos k \cdot\left(x^{(j)}-x^{(l)}\right)}{|k|^{2}} \\
& =\lim _{M_{1} \rightarrow \infty} \lim _{L_{1}, L_{2}, L_{3} \rightarrow \infty} \frac{2 \pi}{|V|} \sum_{k \in \Lambda_{1}} \sum_{j, l=1, j \neq l}^{n} \frac{e_{j} e_{l} \cos k \cdot\left(x^{(j)}-x^{(l)}\right)}{|k|^{2}} \\
& =\frac{1}{2} \sum_{j, l=1, j \neq l}^{n} \frac{e_{j} e_{l}}{\left|x^{(j)}-x^{(l)}\right|} \text { in } \mathcal{S}^{\prime}\left(R^{3 n}\right) .
\end{aligned}
$$


Let $\chi_{0}(k)$ be the function in $R^{3}$ defined by

$$
\chi_{0}(k):= \begin{cases}1, & |k| \leq 1 \\ 0, & |k|>1\end{cases}
$$

We first prove

Lemma 4.2. Let $\epsilon>0$. Then we have

$$
\begin{aligned}
& \lim _{\epsilon \rightarrow 0} \frac{1}{(2 \pi)^{2}} \sum_{j, l=1, j \neq l}^{n} e_{j} e_{l} \int \frac{\cos k \cdot\left(x^{(j)}-x^{(l)}\right)}{|k|^{2}} \chi_{0}(\epsilon k) d k \\
& =\frac{1}{2} \sum_{j, l=1, j \neq l}^{n} \frac{e_{j} e_{l}}{\left|x^{(j)}-x^{(l)}\right|} \text { in } \mathcal{S}^{\prime}\left(R^{3 n}\right) .
\end{aligned}
$$

Proof. Let $x$ and $k$ be in $R^{3}$. Then, it is well known that

$$
\frac{1}{(2 \pi)^{2}} \int \frac{\mathrm{e}^{i k \cdot x}}{|k|^{2}} d k=\frac{1}{2|x|} \quad \text { in } \mathcal{S}^{\prime}\left(R^{3}\right)
$$

(cf. $§ 5.9$ in [25]).

For the sake of simplicity we consider the case $n=2$. Let $x=x^{(1)}$ and $y=x^{(2)}$. We will prove

$$
\lim _{\epsilon \rightarrow 0} \frac{1}{(2 \pi)^{2}} \int \frac{\mathrm{e}^{i k \cdot(x-y)}}{|k|^{2}} \chi_{0}(\epsilon k) d k=\frac{1}{2|x-y|} \quad \text { in } \mathcal{S}^{\prime}\left(R^{6}\right)
$$

Let $\varphi(x, y) \in \mathcal{S}\left(R^{6}\right)$. Then, with $<\cdot, \cdot>$ understood as distributional pairing, from (4.6) we have

$$
\begin{aligned}
& \lim _{\epsilon \rightarrow 0}\left\langle\frac{1}{(2 \pi)^{2}} \int \frac{\mathrm{e}^{i k \cdot(x-y)}}{|k|^{2}} \chi_{0}(\epsilon k) d k, \varphi(x, y)\right\rangle \\
& =\lim _{\epsilon \rightarrow 0}\left\{\left\langle\frac{1}{(2 \pi)^{2}} \int \frac{\cos k \cdot(x-y)}{|k|^{2}} \chi_{0}(\epsilon k) d k, \varphi(x, y)\right\rangle\right. \\
& \left.\quad+i\left\langle\frac{1}{(2 \pi)^{2}} \int \frac{\sin k \cdot(x-y)}{|k|^{2}} \chi_{0}(\epsilon k) d k, \varphi(x, y)\right\rangle\right\} \\
& =\lim _{\epsilon \rightarrow 0}\left\langle\frac{1}{(2 \pi)^{2}} \int \frac{\cos k \cdot(x-y)}{|k|^{2}} \chi_{0}(\epsilon k) d k, \varphi(x, y)\right\rangle \\
& =\left\langle\frac{1}{2|x-y|}, \varphi(x, y)\right\rangle .
\end{aligned}
$$


Consequently we obtain (4.4).

The equation (4.6) is equivalent to

$$
\begin{aligned}
& \lim _{\epsilon \rightarrow 0}\left\langle\frac{1}{2 \pi^{2}} \int \frac{\mathrm{e}^{i k \cdot(x-y)}}{|k|^{2}} \chi_{0}(\epsilon k) d k, \varphi(x, y)\right\rangle \\
& =\iint \frac{1}{|x-y|} \varphi(x, y) d x d y
\end{aligned}
$$

for all $\varphi(x, y) \in \mathcal{S}\left(R^{6}\right)$. We set $x^{\prime}=(x-y) / \sqrt{2}$ and $y^{\prime}=(x+y) / \sqrt{2}$. Let $\psi_{1}\left(x^{\prime}\right)$ and $\psi_{2}\left(y^{\prime}\right)$ be in $\mathcal{S}\left(R^{3}\right)$. We take $\varphi(x, y)=\tilde{\varphi}\left(x^{\prime}, y^{\prime}\right):=\psi_{1}\left(x^{\prime}\right) \psi_{2}\left(y^{\prime}\right)$ in the lhs of (4.7). Then the lhs of (4.7) is equal to

$$
\begin{aligned}
& \lim _{\epsilon \rightarrow 0} \frac{1}{2 \pi^{2}} \iiint \frac{\mathrm{e}^{i k \cdot(x-y)}}{|k|^{2}} \chi_{0}(\epsilon k) \psi_{1}\left(x^{\prime}\right) \psi_{2}\left(y^{\prime}\right) d k d x^{\prime} d y^{\prime} \\
& =\lim _{\epsilon \rightarrow 0} \frac{1}{2 \pi^{2}} \int \psi_{1}\left(x^{\prime}\right) d x^{\prime} \int \frac{\mathrm{e}^{i k \cdot \sqrt{2} x^{\prime}}}{|k|^{2}} \chi_{0}(\epsilon k) d k \int \psi_{2}\left(y^{\prime}\right) d y^{\prime},
\end{aligned}
$$

which is also equal to

$$
\int \frac{1}{\sqrt{2}\left|x^{\prime}\right|} \psi_{1}\left(x^{\prime}\right) d x^{\prime} \int \psi_{2}\left(y^{\prime}\right) d y^{\prime}=\iint \frac{\tilde{\varphi}\left(x^{\prime}, y^{\prime}\right)}{\sqrt{2}\left|x^{\prime}\right|} d x^{\prime} d y^{\prime}=\iint \frac{\varphi(x, y)}{|x-y|} d x d y
$$

from (4.5). So, (4.7) holds for $\varphi(x, y)=\psi_{1}\left(x^{\prime}\right) \psi_{2}\left(y^{\prime}\right)$. Since the set of all linear combinations of $\psi_{1}\left(x^{\prime}\right) \psi_{2}\left(y^{\prime}\right)$ for all $\psi_{1}$ and $\psi_{2}$ in $\mathcal{S}\left(R^{3}\right)$ is dense in $\mathcal{S}\left(R_{x^{\prime}, y^{\prime}}^{6}\right)$, so (4.7) holds for all $\varphi(x, y) \in \mathcal{S}\left(R^{6}\right)$. Hence we get (4.6).

Proposition 4.3. Let $c \geq 0$ be a constant. Let $\Phi(k)$ be continuous in $R^{3} \backslash(\{0\} \cup\{|k|=c\})$. We suppose $|\Phi(k)| \leq \phi(|k|)\left(k \in R^{3}\right)$. We assume that $\phi(r)$ is non-increasing in $(0, \infty)$ and that $r^{2} \phi(r)$ is in $L^{1}([0, \infty))$ and is bounded in $(0, \infty)$. Then, $\left((2 \pi)^{3} /|V|\right) \sum_{k \neq 0} \Phi(k)$ is absolutely convergent, where the sum of $k$ is taken over $\left(2 \pi s_{1} / L_{1}, 2 \pi s_{2} / L_{2}, 2 \pi s_{3} / L_{3}\right)\left(s_{1}, s_{2}, s_{3}=\right.$ $0, \pm 1, \pm 2, \ldots)$. We also get

$$
\lim _{L_{1}, L_{2}, L_{3} \rightarrow \infty} \frac{(2 \pi)^{3}}{|V|} \sum_{k \neq 0} \Phi(k)=\int \Phi(k) d k
$$

under the condition (4.1). 
Proof. We write $L=\left(L_{1}, L_{2}, L_{3}\right)$. Let us define the step function $\Phi_{L}(k)$ by

$$
\begin{aligned}
\Phi_{L}(k) & =\Phi\left(\frac{2 \pi s_{1}}{L_{1}}, \frac{2 \pi s_{2}}{L_{2}}, \frac{2 \pi s_{3}}{L_{3}}\right), \quad k \in\left(\frac{2 \pi\left(s_{1}-1\right)}{L_{1}}, \frac{2 \pi s_{1}}{L_{1}}\right] \\
\times & \left(\frac{2 \pi\left(s_{2}-1\right)}{L_{2}}, \frac{2 \pi s_{2}}{L_{2}}\right] \times\left(\frac{2 \pi\left(s_{3}-1\right)}{L_{3}}, \frac{2 \pi s_{3}}{L_{3}}\right], \\
\Phi_{L}(k) & =\Phi\left(\frac{2 \pi s_{1}}{L_{1}},-\frac{2 \pi s_{2}}{L_{2}}, \frac{2 \pi s_{3}}{L_{3}}\right), \quad k \in\left(\frac{2 \pi\left(s_{1}-1\right)}{L_{1}}, \frac{2 \pi s_{1}}{L_{1}}\right] \\
\times & {\left[-\frac{2 \pi s_{2}}{L_{2}},-\frac{2 \pi\left(s_{2}-1\right)}{L_{2}}\right) \times\left(\frac{2 \pi\left(s_{3}-1\right)}{L_{3}}, \frac{2 \pi s_{3}}{L_{3}}\right] }
\end{aligned}
$$

for $s_{1}, s_{2}, s_{3}=1,2, \ldots$ Then, for $k \in\left(2 \pi\left(s_{1}-1\right) / L_{1}, 2 \pi s_{1} / L_{1}\right] \times\left(2 \pi\left(s_{2}-\right.\right.$ 1) $\left./ L_{2}, 2 \pi s_{2} / L_{2}\right] \times\left(2 \pi\left(s_{3}-1\right) / L_{3}, 2 \pi s_{3} / L_{3}\right]$ we have

$$
\begin{aligned}
\left|\Phi_{L}(k)\right| & =\left|\Phi\left(\frac{2 \pi s_{1}}{L_{1}}, \frac{2 \pi s_{2}}{L_{2}}, \frac{2 \pi s_{3}}{L_{3}}\right)\right| \\
& \leq \phi\left(\left|\left(\frac{2 \pi s_{1}}{L_{1}}, \frac{2 \pi s_{2}}{L_{2}}, \frac{2 \pi s_{3}}{L_{3}}\right)\right|\right) \leq \phi(|k|)
\end{aligned}
$$

since $\phi(r)$ is non-increasing. In the same way, for $k \in\left(2 \pi\left(s_{1}-1\right) / L_{1}, 2 \pi s_{1} / L_{1}\right] \times$ $\left[-2 \pi s_{2} / L_{2},-2 \pi\left(s_{2}-1\right) / L_{2}\right) \times\left(2 \pi\left(s_{3}-1\right) / L_{3}, 2 \pi s_{3} / L_{3}\right]$ we get

$$
\left|\Phi_{L}(k)\right| \leq \phi(|k|)
$$

In the same way as in the above we can define the step function $\Phi_{L}(k)$ for all $k \in R^{3} \backslash\{0\}$ such that (4.9) and

$$
\frac{(2 \pi)^{3}}{|V|} \sum_{k \neq 0} \Phi(k)=\int_{R^{3}} \Phi_{L}(k) d k+\frac{(2 \pi)^{3}}{|V|} \sum_{k \neq 0, s_{1} s_{2} s_{3}=0} \Phi(k) .
$$

For a short while we suppose $L_{1} \leq L_{2} \leq L_{3}$. Since $\phi(r)$ is non-increasing, it holds that for $s_{1} \geq 2$ we have

$$
\begin{gathered}
\phi\left(\left|\left(\frac{2 \pi s_{1}}{L_{1}}, 0,0\right)\right|\right) \leq \phi\left(\left|\left(\frac{2 \pi\left(s_{1}-1\right)}{L_{1}}, \frac{2 \pi}{L_{2}}, \frac{2 \pi}{L_{3}}\right)\right|\right) \leq \phi(|k|), \\
k \in\left(\frac{2 \pi\left(s_{1}-2\right)}{L_{1}}, \frac{2 \pi\left(s_{1}-1\right)}{L_{1}}\right] \times\left(0, \frac{2 \pi}{L_{2}}\right] \times\left(0, \frac{2 \pi}{L_{3}}\right]
\end{gathered}
$$


and also for $s_{1} \geq 2$ and $s_{2} \geq 1$

$$
\begin{gathered}
\phi\left(\left|\left(\frac{2 \pi s_{1}}{L_{1}}, \frac{2 \pi s_{2}}{L_{2}}, 0\right)\right|\right) \leq \phi\left(\left|\left(\frac{2 \pi\left(s_{1}-1\right)}{L_{1}}, \frac{2 \pi s_{2}}{L_{2}}, \frac{2 \pi}{L_{3}}\right)\right|\right) \leq \phi(|k|), \\
k \in\left(\frac{2 \pi\left(s_{1}-2\right)}{L_{1}}, \frac{2 \pi\left(s_{1}-1\right)}{L_{1}}\right] \times\left(\frac{2 \pi\left(s_{2}-1\right)}{L_{2}}, \frac{2 \pi s_{2}}{L_{2}}\right] \times\left(0, \frac{2 \pi}{L_{3}}\right] .
\end{gathered}
$$

For $s_{2} \geq 2$ we also have

$$
\begin{gathered}
\phi\left(\left|\left(\frac{2 \pi}{L_{1}}, \frac{2 \pi s_{2}}{L_{2}}, 0\right)\right|\right) \leq \phi\left(\left|\left(\frac{2 \pi}{L_{1}}, \frac{2 \pi\left(s_{2}-1\right)}{L_{2}}, \frac{2 \pi}{L_{3}}\right)\right|\right) \leq \phi(|k|), \\
k \in\left(0, \frac{2 \pi}{L_{1}}\right] \times\left(\frac{2 \pi\left(s_{2}-2\right)}{L_{2}}, \frac{2 \pi\left(s_{2}-1\right)}{L_{2}}\right] \times\left(0, \frac{2 \pi}{L_{3}}\right] .
\end{gathered}
$$

Thus we get

$$
\begin{aligned}
& \frac{(2 \pi)^{3}}{|V|} \sum_{k \neq 0, s_{3}=0}|\Phi(k)| \leq \frac{(2 \pi)^{3}}{|V|} \sum_{k \neq 0, s_{3}=0} \phi(|k|) \leq \frac{(2 \pi)^{3}}{|V|} \sum_{k \neq 0, s_{3}=0, s_{1}, s_{2}=0, \pm 1} \phi(|k|) \\
& +\frac{(2 \pi)^{3}}{|V|} \sum_{k \neq 0, s_{3}=s_{1}=0,\left|s_{2}\right| \geq 2} \phi(|k|)+10 \int_{0 \leq k_{3} \leq(2 \pi) / L_{3}} \phi(|k|) d k .
\end{aligned}
$$

We can take a constant $1 \leq m \leq m_{0}$ from (4.1) such that $L_{2} \leq m L_{1} \leq L_{3}$. We add the refinement $\left\{\left((2 \pi) /\left(m L_{1}\right),\left(2 \pi s_{2}\right) / L_{2},\left(2 \pi s_{3}\right) / L_{3}\right) ; s_{2}, s_{3}=0, \pm 1, \pm 2, \ldots\right\}$ to $\left\{\left(\left(2 \pi s_{1}\right) / L_{1},\left(2 \pi s_{2}\right) / L_{2},\left(2 \pi s_{3}\right) / L_{3}\right) ; s_{1}, s_{2}, s_{3}=0, \pm 1, \pm 2, \ldots\right\}$. Then, for $s_{2} \geq 2$ noting

$$
\begin{gathered}
\phi\left(\left|\left(0, \frac{2 \pi s_{2}}{L_{2}}, 0\right)\right|\right) \leq \phi\left(\left|\left(\frac{2 \pi}{m L_{1}}, \frac{2 \pi\left(s_{2}-1\right)}{L_{2}}, \frac{2 \pi}{L_{3}}\right)\right|\right) \leq \phi(|k|), \\
k \in\left(0, \frac{2 \pi}{m L_{1}}\right] \times\left(\frac{2 \pi\left(s_{2}-2\right)}{L_{2}}, \frac{2 \pi\left(s_{2}-1\right)}{L_{2}}\right] \times\left(0, \frac{2 \pi}{L_{3}}\right],
\end{gathered}
$$

we have

$$
\begin{aligned}
\frac{(2 \pi)^{3}}{m|V|} \sum_{k \neq 0, s_{3}=s_{1}=0,\left|s_{2}\right| \geq 2} \phi(|k|) & \leq 2 \int_{0 \leq k_{1} \leq(2 \pi) /\left(m L_{1}\right), 0 \leq k_{3} \leq(2 \pi) / L_{3}} \phi(|k|) d k \\
& \leq 2 \int_{0 \leq k_{3} \leq(2 \pi) / L_{3}} \phi(|k|) d k .
\end{aligned}
$$


Consequently from (4.11) we get

$$
\begin{aligned}
& \frac{(2 \pi)^{3}}{|V|} \sum_{k \neq 0, s_{3}=0}|\Phi(k)| \leq \frac{(2 \pi)^{3}}{|V|} \sum_{k \neq 0, s_{3}=0, s_{1}, s_{2}=0, \pm 1} \phi(|k|) \\
& \quad+2\left(5+m_{0}\right) \int_{0 \leq k_{3} \leq(2 \pi) / L_{3}} \phi(|k|) d k .
\end{aligned}
$$

Let us consider the case of general $L_{1}, L_{2}$ and $L_{3}$. We may suppose $L_{1} \leq L_{2}$. Noting $L_{2} \leq m_{0} L_{3}$ from (4.1), we add the refinement $\left\{\left(\left(2 \pi s_{1}\right) / L_{1},\left(2 \pi s_{2}\right) / L_{2}\right.\right.$, $\left.\left.(2 \pi) /\left(m_{0} L_{3}\right)\right) ; s_{1}, s_{2}=0, \pm 1, \pm 2, \ldots\right\}$ to $\left\{\left(\left(2 \pi s_{1}\right) / L_{1},\left(2 \pi s_{2}\right) / L_{2},\left(2 \pi s_{3}\right) / L_{3}\right)\right.$; $\left.s_{1}, s_{2}, s_{3}=0, \pm 1, \pm 2, \ldots\right\}$. Then, as in the proof to (4.11), for $s_{1} \geq 2$ we have

$$
\begin{gathered}
\phi\left(\left|\left(\frac{2 \pi s_{1}}{L_{1}}, 0,0\right)\right|\right) \leq \phi\left(\left|\left(\frac{2 \pi\left(s_{1}-1\right)}{L_{1}}, \frac{2 \pi}{L_{2}}, \frac{2 \pi}{m_{0} L_{3}}\right)\right|\right) \leq \phi(|k|), \\
k \in\left(\frac{2 \pi\left(s_{1}-2\right)}{L_{1}}, \frac{2 \pi\left(s_{1}-1\right)}{L_{1}}\right] \times\left(0, \frac{2 \pi}{L_{2}}\right] \times\left(0, \frac{2 \pi}{m_{0} L_{3}}\right]
\end{gathered}
$$

and also for $s_{1} \geq 2$ and $s_{2} \geq 1$

$$
\begin{array}{r}
\phi\left(\left|\left(\frac{2 \pi s_{1}}{L_{1}}, \frac{2 \pi s_{2}}{L_{2}}, 0\right)\right|\right) \leq \phi\left(\left|\left(\frac{2 \pi\left(s_{1}-1\right)}{L_{1}}, \frac{2 \pi s_{2}}{L_{2}}, \frac{2 \pi}{m_{0} L_{3}}\right)\right|\right) \leq \phi(|k|), \\
k \in\left(\frac{2 \pi\left(s_{1}-2\right)}{L_{1}}, \frac{2 \pi\left(s_{1}-1\right)}{L_{1}}\right] \times\left(\frac{2 \pi\left(s_{2}-1\right)}{L_{2}}, \frac{2 \pi s_{2}}{L_{2}}\right] \times\left(0, \frac{2 \pi}{m_{0} L_{3}}\right] .
\end{array}
$$

For $s_{2} \geq 2$ we also have

$$
\begin{gathered}
\phi\left(\left|\left(\frac{2 \pi}{L_{1}}, \frac{2 \pi s_{2}}{L_{2}}, 0\right)\right|\right) \leq \phi\left(\left|\left(\frac{2 \pi}{L_{1}}, \frac{2 \pi\left(s_{2}-1\right)}{L_{2}}, \frac{2 \pi}{m_{0} L_{3}}\right)\right|\right) \leq \phi(|k|), \\
k \in\left(0, \frac{2 \pi}{L_{1}}\right] \times\left(\frac{2 \pi\left(s_{2}-2\right)}{L_{2}}, \frac{2 \pi\left(s_{2}-1\right)}{L_{2}}\right] \times\left(0, \frac{2 \pi}{m_{0} L_{3}}\right] .
\end{gathered}
$$

Hence we can prove

$$
\begin{aligned}
& \frac{(2 \pi)^{3}}{|V|} \sum_{k \neq 0, s_{3}=0}|\Phi(k)| \leq m_{0} \frac{(2 \pi)^{3}}{m_{0}|V|} \sum_{k \neq 0, s_{3}=0} \phi(|k|) \leq \frac{(2 \pi)^{3}}{|V|} \sum_{k \neq 0, s_{3}=0, s_{1}, s_{2}=0, \pm 1} \phi(|k|) \\
& \quad+\frac{(2 \pi)^{3}}{|V|} \sum_{k \neq 0, s_{3}=s_{1}=0,\left|s_{2}\right| \geq 2} \phi(|k|)+10 m_{0} \int_{0 \leq k_{3} \leq(2 \pi) /\left(m_{0} L_{3}\right)} \phi(|k|) d k
\end{aligned}
$$


as in the proof of (4.11) and so

$$
\begin{aligned}
& \frac{(2 \pi)^{3}}{|V|} \sum_{k \neq 0, s_{3}=0}|\Phi(k)| \leq \frac{(2 \pi)^{3}}{|V|} \sum_{k \neq 0, s_{3}=0, s_{1}, s_{2}=0, \pm 1} \phi(|k|) \\
& \quad+2 m_{0}\left(5+m_{0}\right) \int_{0 \leq k_{3} \leq(2 \pi) /\left(m_{0} L_{3}\right)} \phi(|k|) d k .
\end{aligned}
$$

as in the proof of (4.12). Thus, for general $L_{1}, L_{2}$ and $L_{3}$ we obtain

$$
\begin{aligned}
& \frac{(2 \pi)^{3}}{|V|} \sum_{k \neq 0, s_{j}=0}|\Phi(k)| \leq \frac{(2 \pi)^{3}}{|V|} \sum_{k \neq 0, s_{1}, s_{2}, s_{3}=0, \pm 1} \phi(|k|) \\
& \quad+2 m_{0}\left(5+m_{0}\right) \int_{0 \leq k_{j} \leq(2 \pi) /\left(m_{0} L_{j}\right)} \phi(|k|) d k \quad(j=1,2,3) .
\end{aligned}
$$

We assumed that $r^{2} \phi(r)$ is in $L^{1}(R)$. So, from (4.9), (4.10) and (4.13) we can prove that $\sum_{k \neq 0}|\Phi(k)|$ is convergent. In addition, since $r^{2} \phi(r)$ is assumed to be bounded in $(0, \infty)$,

$$
0 \leq \phi(|k|) \leq \text { Const. } \frac{1}{|k|^{2}}, \quad k \neq 0
$$

holds. So we see that $\left((2 \pi)^{3} /|V|\right) \sum_{k \neq 0, s_{1}, s_{2}, s_{3}=0, \pm 1} \phi(|k|)$ tends to zero as $L_{1}, L_{2}$ and $L_{3}$ tend to the infinity under the condition (4.1). Consequently from (4.13) we have

$$
\lim _{L_{1}, L_{2}, L_{3} \rightarrow \infty} \frac{(2 \pi)^{3}}{|V|} \sum_{k \neq 0, s_{j}=0} \Phi(k)=0, \quad j=1,2,3
$$

under (4.1). Hence, noting (4.9), from (4.10) we obtain (4.8) by means of the Lebesgue dominated convergence theorem.

Now we will prove Theorem 4.1. For the sake of simplicity let $n=2$. Let $\chi_{0}(k)$ be the function defined by (4.3). We write $x=x^{(1)}$ and $y=x^{(2)}$. We 
take $\varphi(x, y) \in \mathcal{S}\left(R^{6}\right)$. Then, we have

$$
\begin{aligned}
& \left\langle\frac{(2 \pi)^{3}}{|V|} \sum_{k \neq 0} \frac{\cos k \cdot(x-y)}{|k|^{2}} \chi_{0}(\epsilon k), \varphi(x, y)\right\rangle \\
& =\frac{(2 \pi)^{3}}{|V|} \sum_{k \neq 0} \iint \frac{\cos k \cdot(x-y)}{|k|^{2}} \chi_{0}(\epsilon k) \varphi(x, y) d x d y \\
& =\frac{(2 \pi)^{3}}{|V|} \sum_{k \neq 0} \iint \frac{\cos k \cdot(x-y)}{|k|^{2}<k>^{2}} \chi_{0}(\epsilon k)<D_{x}>^{2} \varphi(x, y) d x d y,
\end{aligned}
$$

where we define $<D_{x}>^{2}:=\left(1-\sum_{j=1}^{n} \partial_{x_{j}}^{2}\right)$. Let $\Phi(k)=|k|^{-2}<k>^{-2}$ $\times \iint \cos k \cdot(x-y)<D_{x}>^{2} \varphi(x, y) d x d y$ and

$$
\phi(|k|):=\frac{1}{|k|^{2}<k>^{2}} \iint\left|<D_{x}>^{2} \varphi(x, y)\right| d x d y .
$$

Then from (4.14) Proposition 4.3 shows

$$
\begin{aligned}
& \lim _{L_{1}, L_{2}, L_{3} \rightarrow \infty} \lim _{\epsilon \rightarrow 0}\left\langle\frac{(2 \pi)^{3}}{|V|} \sum_{k \neq 0} \frac{\cos k \cdot(x-y)}{|k|^{2}} \chi_{0}(\epsilon k), \varphi(x, y)\right\rangle \\
& =\lim _{L_{1}, L_{2}, L_{3} \rightarrow \infty} \frac{(2 \pi)^{3}}{|V|} \sum_{k \neq 0} \iint \frac{\cos k \cdot(x-y)}{|k|^{2}<k>^{2}}<D_{x}>^{2} \varphi(x, y) d x d y \\
& =\int \frac{1}{|k|^{2}<k>^{2}} d k \iint(\cos k \cdot(x-y))<D_{x}>^{2} \varphi(x, y) d x d y .
\end{aligned}
$$

In the same way from (4.14) we also have

$$
\begin{aligned}
& \lim _{\epsilon \rightarrow 0} \lim _{L_{1}, L_{2}, L_{3} \rightarrow \infty}\left\langle\frac{(2 \pi)^{3}}{|V|} \sum_{k \neq 0} \frac{\cos k \cdot(x-y)}{|k|^{2}} \chi_{0}(\epsilon k), \varphi(x, y)\right\rangle \\
& =\int \frac{1}{|k|^{2}<k>^{2}} d k \iint(\cos k \cdot(x-y))<D_{x}>^{2} \varphi(x, y) d x d y .
\end{aligned}
$$


On the other hand, Lemma 4.2 and Proposition 4.3 indicate

$$
\begin{aligned}
& \lim _{\epsilon \rightarrow 0} \lim _{L_{1}, L_{2}, L_{3} \rightarrow \infty}\left\langle\frac{(2 \pi)^{3}}{|V|} \sum_{k \neq 0} \frac{\cos k \cdot(x-y)}{|k|^{2}} \chi_{0}(\epsilon k), \varphi(x, y)\right\rangle \\
& =\lim _{\epsilon \rightarrow 0} \iiint \frac{\cos k \cdot(x-y)}{|k|^{2}} \chi_{0}(\epsilon k) \varphi(x, y) d x d y d k \\
& =2 \pi^{2} \iint \frac{\varphi(x, y)}{|x-y|} d x d y .
\end{aligned}
$$

Hence we obtain (4.2) together with (4.15) and (4.16).

Remark 4.1. Let $\chi(k) \in \mathcal{S}\left(R^{3}\right)$ such that $\chi(0)=1$ and $\chi(-k)=\chi(k)$. We take the limit of $L_{j}(j=1,2,3)$ under the condition (4.1). Then it holds that

$$
\begin{aligned}
& \lim _{\epsilon \rightarrow 0} \lim _{L_{1}, L_{2}, L_{3} \rightarrow \infty} \frac{2 \pi}{|V|} \sum_{k \neq 0} \sum_{j, l=1, j \neq l}^{n} \chi(\epsilon k) \frac{e_{j} e_{l} \cos k \cdot\left(x^{(j)}-x^{(l)}\right)}{|k|^{2}} \\
& =\frac{1}{2} \sum_{j, l=1, j \neq l}^{n} \frac{e_{j} e_{l}}{\left|x^{(j)}-x^{(l)}\right|}
\end{aligned}
$$

pointwise for $\vec{x} \in R^{3 n}$ such that $x^{(j)}-x^{(l)} \neq 0(j, l=1,2, \ldots, n, j \neq l)$. The proof is easy. Consider the case $n=2$ and $e_{1}=e_{2}=1$. Let us write $x=x^{(1)}$ and $y=x^{(2)}$. We take $\chi_{1}(k) \in C^{\infty}\left(R^{3}\right)$ such that $\chi_{1}(k)=1(|k| \leq 1)$ and $\chi_{1}(k)=0(|k| \geq 2)$. Then, Proposition 4.3 says for $x \neq y$ that the lhs of (4.18) is equal to

$$
\begin{aligned}
& \frac{1}{(2 \pi)^{2}} \lim _{\epsilon \rightarrow 0} \int \chi(\epsilon k) \frac{\cos k \cdot(x-y)}{|k|^{2}} d k \\
& =\frac{1}{(2 \pi)^{2}} \lim _{\epsilon \rightarrow 0}\left\{\int \chi_{1}(k) \chi(\epsilon k) \frac{\cos k \cdot(x-y)}{|k|^{2}} d k\right. \\
& \left.-\frac{1}{|x-y|^{2}} \int(\cos k \cdot(x-y)) \Delta_{k}\left\{\left(1-\chi_{1}(k)\right) \chi(\epsilon k)|k|^{-2}\right\} d k\right\} \\
& =\frac{1}{(2 \pi)^{2}}\left\{\int \chi_{1}(k) \frac{\cos k \cdot(x-y)}{|k|^{2}} d k\right. \\
& \left.-\frac{1}{|x-y|^{2}} \int(\cos k \cdot(x-y)) \Delta_{k}\left\{\left(1-\chi_{1}(k)\right)|k|^{-2}\right\} d k\right\}
\end{aligned}
$$

pointwise, where $\Delta_{k}$ denotes the Laplacian operator with respect to $k \in R^{3}$ and we used $\left|\epsilon \chi^{\prime}(\epsilon k)\right|=\epsilon^{1 / 3}|k|^{-2 / 3}(\epsilon|k|)^{2 / 3}\left|\chi^{\prime}(\epsilon k)\right| \leq$ Const. $\epsilon^{1 / 3}|k|^{-2 / 3}$. Since 
we have $\left|\Delta_{k}\left\{\left(1-\chi_{1}(k)\right) \chi(\epsilon k)|k|^{-2}\right\}\right| \leq C<k>^{-3-1 / 3}$ with a constant $C$ independent of $\epsilon$, so we can prove that the equation (4.19) is also true in the distribution sense $\mathcal{S}^{\prime}\left(R^{6}\right)$. On the other hand, we see as in the proof of Lemma 4.2 that the lhs of $(4.19)$ is equal to $1 /(2|x-y|)$ in $\mathcal{S}^{\prime}\left(R^{6}\right)$. Consequently we can prove that $(4.19)$ is equal to $1 /(2|x-y|)$. Hence (4.18) holds pointwise.

\section{The expression for the vacuum and the states of photons}

In this section we express the vacuum and the states of photons with given momenta and polarizations concretely as functions of variables $a_{\Lambda^{\prime}}$ consisting of the Fourier coefficients of vector potentials. In Problem 9-8 of [11] only the vacuum and the state of a photon of momentum $h k$ and polarization state 1 are expressed concretely. In this section we generalize this result in [11] for the general states of photons. In physics the vacuum and the state of photons are not considered concretely but rather considered abstractly (cf. [29, 33]). We also note that the state of photons of given momenta and polarizations are not discussed in the study for QED models defined by means of the functional method (cf. [12, 14, 16, 32]), because in the functional method each photon with polarizations is expressed by an amplitude of momentum in $L^{2}\left(R^{3}\right) \oplus$ $L^{2}\left(R^{3}\right)$ as stated in the introduction.

To write down the vacuum and the state of photons concretely, we will introduce creation operators and annihilation operators acting on the space $\mathcal{S}^{\prime}\left(R_{a_{\Lambda^{\prime}}}^{4 N}\right)$. Let us define

$$
\begin{aligned}
\hat{a}_{1 k}^{(\mathrm{i})} & :=i \sqrt{\frac{|V|}{2 h c|k|}}\left(\frac{h}{i} \frac{\partial}{\partial a_{1 k}^{(\mathrm{i})}}-i \frac{c|k|}{|V|} a_{\mathrm{l} k}^{(\mathrm{i})}\right) \\
& =\sqrt{\frac{|V|}{2 h c|k|}}\left(h \frac{\partial}{\partial a_{\mathrm{l} k}^{(\mathrm{i})}}+\frac{c|k|}{|V|} a_{1 k}^{(\mathrm{i})}\right)
\end{aligned}
$$

acting on the space $\mathcal{S}^{\prime}\left(R_{a_{\Lambda^{\prime}}}^{4 N}\right)$ for $k \in \Lambda$ and i,l $=1,2$. From (2.13) we have

$$
\hat{a}_{1-k}^{(1)}=-\hat{a}_{1 k}^{(1)}, \hat{a}_{1-k}^{(2)}=\hat{a}_{1 k}^{(2)} .
$$


Let $\hat{a}_{1 k}^{(\mathrm{i}) \dagger}$ denote the formal adjoint operator $\sqrt{|V| /(2 h c|k|)}\left(-h \partial / \partial a_{1 k}^{(\mathrm{i})}+c|k| a_{1 k}^{(\mathrm{i})} /|V|\right)$ of $\hat{a}_{1 k}^{(\mathrm{i})}$ acting on the space $\mathcal{S}^{\prime}\left(R_{a_{\Lambda^{\prime}}}^{4 N}\right)$. For $f \in \mathcal{S}^{\prime}\left(R_{a_{\Lambda^{\prime}}}^{4 N}\right)$ and $g \in \mathcal{S}\left(R_{a_{\Lambda^{\prime}}}^{4 N}\right)$ we have

$$
\left(\hat{a}_{1 k}^{(\mathrm{i})} f, g\right)=\left(f, \hat{a}_{1 k}^{(\mathrm{i}) \dagger} g\right)
$$

from the definition of the distribution. So, $\hat{a}_{1 k}^{(\mathrm{i})}$ is continuous from $\mathcal{S}^{\prime}\left(R_{a_{\Lambda^{\prime}}}^{4 N}\right)$ into $\mathcal{S}^{\prime}\left(R_{a_{\Lambda^{\prime}}}^{4 N}\right)$ in weak topology. In the same way $\hat{a}_{1 k}^{(\mathrm{i}) \dagger}$ is continuous from $\mathcal{S}^{\prime}\left(R_{a_{\Lambda^{\prime}}}^{4 N}\right)$ into $\mathcal{S}^{\prime}\left(R_{a_{\Lambda^{\prime}}}^{4 N}\right)$ in weak topology.

We can easily see from (5.1) that the commutator relations

$$
\left[\hat{a}_{1 k}^{(\mathrm{i})}, \hat{a}_{1^{\prime} k^{\prime}}^{\left(\mathrm{i}^{\prime}\right) \dagger}\right]=\delta_{\mathrm{i}^{\prime} \mathrm{i}} \delta_{\mathrm{l}^{\prime} \mathrm{l}} \delta_{k k^{\prime}}, \quad\left[\hat{a}_{\mathrm{l} k}^{(\mathrm{i})}, \hat{a}_{\mathrm{l}^{\prime} k^{\prime}}^{\left(\mathrm{i}^{\prime}\right)}\right]=0
$$

on $\mathcal{S}\left(R_{a_{\Lambda^{\prime}}}^{4 N}\right)$ and so on $\mathcal{S}^{\prime}\left(R_{a_{\Lambda^{\prime}}}^{4 N}\right)$ hold for $k$ and $k^{\prime}$ in the bounded domain $\Lambda^{\prime}$ (cf. $\S 34$ in [7] and [6, 30]). For $\mathcal{S}\left(R_{a_{\Lambda^{\prime}}}^{4 N}\right)$ is dense in $\mathcal{S}^{\prime}\left(R_{a_{\Lambda^{\prime}}}^{4 N}\right)$ in weak topology (cf. [26]) and the operators of both sides above are continuous in $\mathcal{S}^{\prime}\left(R_{a_{\Lambda^{\prime}}}^{4 N}\right)$. We define the operator $\hat{a}_{\mathrm{l} k}$ acting on $\mathcal{S}^{\prime}\left(R_{a_{\Lambda^{\prime}}}^{4 N}\right)$ for $k \in \Lambda$ and $\mathrm{l}=1,2$ by

$$
\hat{a}_{\mathrm{lk}}:=\frac{\hat{a}_{1 k}^{(1)}-i \hat{a}_{1 k}^{(2)}}{\sqrt{2}}
$$

(cf. (2.11)). We call $\hat{a}_{1 k}$ the annihilation operator and $\hat{a}_{1 k}^{\dagger}$ the creation operator. We can easily see from the commutator relations for $\hat{a}_{1 k}^{(\mathrm{i})}$ that the operators $\hat{a}_{1 k}$ and $\hat{a}_{l k}^{\dagger}$ also satisfy the commutator relations

$$
\left[\hat{a}_{1 k}, \hat{a}_{1^{\prime} k^{\prime}}^{\dagger}\right]=\delta_{\mathrm{l}^{\prime} 1} \delta_{k k^{\prime}}, \quad\left[\hat{a}_{\mathrm{l} k}, \hat{a}_{\mathrm{l}^{\prime} k^{\prime}}\right]=0
$$

on $\mathcal{S}^{\prime}\left(R_{a_{\Lambda^{\prime}}}^{4 N}\right)$ for $k$ and $k^{\prime}$ in $\Lambda$ (cf. (2.26) in [29]). It follows from the commutator relations (5.3) that we have

$$
\hat{a}_{1 k}\left(\hat{a}_{1 k}^{\dagger}\right)^{n^{\prime}}-\left(\hat{a}_{1 k}^{\dagger}\right)^{n^{\prime}} \hat{a}_{1 k}=n^{\prime}\left(\hat{a}_{1 k}^{\dagger}\right)^{n^{\prime}-1}
$$

on $\mathcal{S}^{\prime}\left(R_{a_{\Lambda^{\prime}}}^{4 N}\right)$ (cf. $\S 34$ in [7]). Then we get the following expression as in physics (cf. e.g. (2.60) and (2.64) in [29], and (6.165) and (6.172) in [33]).

Proposition 5.1. We can write the last term of $H(t)$ defined by (3.10) as

$$
\begin{aligned}
H_{\text {rad }} & :=\sum_{k \in \Lambda^{\prime}, 1} \sum_{\mathrm{i}=1}^{2}\left\{\frac{|V|}{2}\left(\frac{h}{i} \frac{\partial}{\partial a_{1 k}^{(\mathrm{i})}}\right)^{2}+\frac{(c|k|)^{2}}{2|V|}\left(a_{1 k}^{(\mathrm{i})}\right)^{2}-\frac{h c|k|}{2}\right\} \\
& =\sum_{k \in \Lambda, 1} h c|k| \hat{a}_{\mathrm{l} k}^{\dagger} \hat{a}_{\mathrm{l} k}
\end{aligned}
$$


on $\mathcal{S}^{\prime}\left(R_{a_{\Lambda^{\prime}}}^{4 N}\right)$. The vector potential $A\left(x, a_{\Lambda_{2}^{\prime}}\right)$ defined by $(2.14)$, where the sum of $k$ is taken over $\Lambda_{2}$, is given for each $x \in R^{3}$ by the expression

$$
A\left(x, a_{\Lambda_{2}^{\prime}}\right)=\sqrt{\frac{4 \pi h}{|V|}} c \sum_{k \in \Lambda_{2}} \sum_{\mathrm{l}=1}^{2} \frac{1}{\sqrt{2 c|k|}}\left(\hat{a}_{1 k} \mathrm{e}^{i k \cdot x}+\hat{a}_{1 k}^{\dagger} \mathrm{e}^{-i k \cdot x}\right) \overrightarrow{e_{1}}(k)
$$

acting on $\mathcal{S}^{\prime}\left(R_{a_{\Lambda^{\prime}}}^{4 N}\right)$.

Proof. Since from (5.1) and (5.2) we have

$$
\begin{aligned}
& h c|k|\left(\hat{a}_{1 k}^{\dagger} \hat{a}_{1 k}+\hat{a}_{1-k}^{\dagger} \hat{a}_{1-k}\right) \\
& =\frac{h c|k|}{2}\left\{\left(\hat{a}_{1 k}^{(1) \dagger}+i \hat{a}_{1 k}^{(2) \dagger}\right)\left(\hat{a}_{1 k}^{(1)}-i \hat{a}_{1 k}^{(2)}\right)+\left(-\hat{a}_{1 k}^{(1) \dagger}+i \hat{a}_{1 k}^{(2) \dagger}\right)\left(-\hat{a}_{1 k}^{(1)}-i \hat{a}_{1 k}^{(2)}\right)\right\} \\
& =h c|k|\left(\hat{a}_{1 k}^{(1) \dagger} \hat{a}_{1 k}^{(1)}+\hat{a}_{1 k}^{(2) \dagger} \hat{a}_{1 k}^{(2)}\right) \\
& =\sum_{\mathrm{i}=1}^{2}\left\{\frac{|V|}{2}\left(\frac{h}{i} \frac{\partial}{\partial a_{1 k}^{(\mathrm{i})}}\right)^{2}+\frac{(c|k|)^{2}}{2|V|}\left(a_{1 k}^{(\mathrm{i})}\right)^{2}-\frac{h c|k|}{2}\right\}
\end{aligned}
$$

on $\mathcal{S}\left(R_{a_{\Lambda^{\prime}}}^{4 N}\right)$ for $k \in \Lambda$, so we get $(5.5)$ on $\mathcal{S}^{\prime}\left(R_{a_{\Lambda^{\prime}}}^{4 N}\right)$ as in the same way as before.

From (5.1) and (5.2) we have

$$
\begin{aligned}
& \hat{a}_{1 k} \mathrm{e}^{i k \cdot x}+\hat{a}_{1 k}^{\dagger} \mathrm{e}^{-i k \cdot x} \\
& =\frac{1}{\sqrt{2}}\left\{\left(\hat{a}_{1 k}^{(1)}+\hat{a}_{1 k}^{(1) \dagger}\right) \cos k \cdot x-i\left(\hat{a}_{1 k}^{(2)}-\hat{a}_{1 k}^{(2) \dagger}\right) \cos k \cdot x\right. \\
& \left.+i\left(\hat{a}_{1 k}^{(1)}-\hat{a}_{1 k}^{(1) \dagger}\right) \sin k \cdot x+\left(\hat{a}_{1 k}^{(2)}+\hat{a}_{1 k}^{(2) \dagger}\right) \sin k \cdot x\right\} \\
& =\sqrt{\frac{|V|}{h c|k|}}\left(\frac{c|k|}{|V|} a_{1 k}^{(1)} \cos k \cdot x+\frac{c|k|}{|V|} a_{1 k}^{(2)} \sin k \cdot x\right. \\
& \left.-i \hbar(\cos k \cdot x) \frac{\partial}{\partial a_{1 k}^{(2)}}+i \hbar(\sin k \cdot x) \frac{\partial}{\partial a_{1 k}^{(1)}}\right)
\end{aligned}
$$

on $\mathcal{S}\left(R_{a_{\Lambda^{\prime}}}^{4 N}\right)$. So, it is shown from (2.8) and (2.13) that

$$
\begin{aligned}
& \sum_{k \in \Lambda_{2}} \frac{1}{\sqrt{2 c|k|}}\left(\hat{a}_{1 k} \mathrm{e}^{i k \cdot x}+\hat{a}_{1 k}^{\dagger} \mathrm{e}^{-i k \cdot x}\right) \overrightarrow{e_{1}}(k) \\
& =\sum_{k \in \Lambda_{2}} \frac{1}{\sqrt{2 h|V|}}\left(a_{1 k}^{(1)} \cos k \cdot x+a_{1 k}^{(2)} \sin k \cdot x\right) \overrightarrow{e_{1}}(k)
\end{aligned}
$$


on $\mathcal{S}\left(R_{a_{\Lambda^{\prime}}}^{4 N}\right)$. Hence, we see that the rhs of (5.6) is equal to

$$
\frac{\sqrt{4 \pi}}{|V|} c \sum_{k \in \Lambda_{2}} \sum_{\mathrm{l}=1}^{2} \frac{1}{\sqrt{2}}\left(a_{1 k}^{(1)} \cos k \cdot x+a_{1 k}^{(2)} \sin k \cdot x\right) \overrightarrow{e_{1}}(k)
$$

on $\mathcal{S}^{\prime}\left(R_{a_{\Lambda^{\prime}}}^{4 N}\right)$, which is equal to the lhs of (5.6) from (2.14).

We know

$$
\int_{-\infty}^{\infty} \mathrm{e}^{-a \theta^{2}} d \theta=\sqrt{\frac{\pi}{a}}
$$

for a constant $a>0$. So, we can easily see from (5.2) and (5.5) that

$$
\Psi_{0}\left(a_{\Lambda^{\prime}}\right):=\prod_{k \in \Lambda^{\prime}, 1} \sqrt{\frac{c|k|}{\pi h|V|}} \exp \left\{-\frac{c|k|}{2 h|V|}\left(a_{1 k}^{(1)^{2}}+a_{1 k}^{(2)^{2}}\right)\right\}
$$

is the normal ground state of $H_{\text {rad }}$, called vacuum, whose energy is 0 , i.e.

$$
H_{\mathrm{rad}} \Psi_{0}=0
$$

and that we have

$$
\hat{a}_{1 k}^{\dagger} \Psi_{0}=\sqrt{\frac{2 c|k|}{h|V|}} a_{1 k}^{*} \Psi_{0}, \hat{a}_{1 k} \Psi_{0}=0 \quad(k \in \Lambda)
$$

(cf. $\S 8-1,(9-43)$ and Problem 9-8 in [11]). We know that the eigenvalue 0 of (5.8) is simple in $L^{2}\left(R^{4 N}\right)$ (cf. Theorem 3.4 in Chapter 3 of [4]).

The function $\Psi_{n^{\prime} 1 k}\left(a_{\Lambda^{\prime}}\right):=\left(\hat{a}_{1 k}^{\dagger}\right)^{n^{\prime}} \Psi_{0}\left(a_{\Lambda^{\prime}}\right)\left(k \in \Lambda, n^{\prime}=0,1,2, \ldots\right)$, which can be written concretely from (5.1), (5.2) and (5.7), expresses the state of $n^{\prime}$ photons of momentum $h k$ and polarization state l (cf. $\S 9-2$ in [11] and $\S 2-2$ in [29]) and satisfies

$$
\begin{gathered}
\left(\sum_{k \in \Lambda, 1} \hat{a}_{1 k}^{\dagger} \hat{a}_{1 k}\right) \Psi_{n^{\prime} \mathrm{l}^{\prime} k^{\prime}}=n^{\prime} \Psi_{n^{\prime} \mathrm{l}^{\prime} k^{\prime}} \\
\left(\sum_{k \in \Lambda} h k \hat{a}_{1 k}^{\dagger} \hat{a}_{1 k}\right) \Psi_{n^{\prime} \mathrm{l}^{\prime} k^{\prime}}=n^{\prime}\left(h k^{\prime}\right) \Psi_{n^{\prime} \mathrm{l}^{\prime} k^{\prime}}
\end{gathered}
$$

and

$$
H_{\mathrm{rad}} \Psi_{n^{\prime} 1^{\prime} k^{\prime}}=n^{\prime}\left(h c\left|k^{\prime}\right|\right) \Psi_{n^{\prime} 1^{\prime} k^{\prime}}
$$


from (5.4), (5.5) and (5.9). The operators $\sum_{k \in \Lambda, 1} \hat{a}_{1 k}^{\dagger} \hat{a}_{1 k}$ and $\sum_{k \in \Lambda} h k \hat{a}_{1 k}^{\dagger} \hat{a}_{1 k}$ are called the total number operator and the momentum operator, respectively (cf. [6], and (2.68) and (2.80) in [29]). Let $n^{\prime}(1, k) \geq 0$ be integers. Then $\prod_{k \in \Lambda, 1}\left(\hat{a}_{1 k}^{\dagger}\right)^{n^{\prime}(1, k)} \Psi_{0}\left(a_{\Lambda^{\prime}}\right)$ denotes the state of $n^{\prime}(1, k)$ photons of momentum $h k$ and polarization state 1 in the same way. Setting $\Psi\left(a_{\Lambda^{\prime}}\right)=$ $\prod_{k \in \Lambda, 1}\left(\hat{a}_{1 k}^{\dagger}\right)^{n^{\prime}(1, k)} \Psi_{0}\left(a_{\Lambda^{\prime}}\right)$, we get

$$
\begin{aligned}
\left(\sum_{k \in \Lambda, l} \hat{a}_{1 k}^{\dagger} \hat{a}_{1 k}\right) \Psi & =\left(\sum_{k \in \Lambda, 1} n^{\prime}(1, k)\right) \Psi \\
\left(\sum_{k \in \Lambda} h k \hat{a}_{1 k}^{\dagger} \hat{a}_{1 k}\right) \Psi & =\left(\sum_{k \in \Lambda, 1} n^{\prime}(1, k) h k\right) \Psi
\end{aligned}
$$

and

$$
H_{\mathrm{rad}} \Psi=\left(\sum_{k \in \Lambda, l} n^{\prime}(1, k) h c|k|\right) \Psi
$$

The family

$$
\left\{\prod_{k \in \Lambda^{\prime}, 1, \mathrm{i}}\left(\hat{a}_{1 k}^{(\mathrm{i}) \dagger}\right)^{n^{\prime}(1, k, \mathrm{i})} \Psi_{0}\right\}_{n^{\prime}(1, k, \mathrm{i})=0}^{\infty}
$$

makes a complete orthogonal system in $L^{2}\left(R^{4 N}\right)$ (cf. Theorem 3.1 in Chapter 3 of [4] and $\S 34$ in [7]). We have

$$
\hat{a}_{1 k}^{(1)}=\frac{\hat{a}_{1 k}-\hat{a}_{1-k}}{\sqrt{2}}, \quad \hat{a}_{1 k}^{(2)}=\frac{i\left(\hat{a}_{1 k}+\hat{a}_{1-k}\right)}{\sqrt{2}}
$$

from (2.13) and (5.2). So we see together with (5.4) and the second equation in (5.9) that the family

$$
\left\{\prod_{k \in \Lambda, 1} \frac{1}{\sqrt{n^{\prime}(1, k) !}}\left(\hat{a}_{1 k}^{\dagger}\right)^{n^{\prime}(1, k)} \Psi_{0}\right\}_{n^{\prime}(1, k)=0}^{\infty}
$$

also makes a complete orthonormal system in $L^{2}\left(R^{4 N}\right)$ (cf. $§ 34$ in [7] and 
(2.46) in [29]). For example, we have

$$
\begin{aligned}
\left(\hat{a}_{1 k}^{\dagger} \Psi_{0},\left(\hat{a}_{1 k}^{\dagger}\right)^{2} \Psi_{0}\right) & =\left(\Psi_{0}, \hat{a}_{1 k}\left(\hat{a}_{1 k}^{\dagger}\right)^{2} \Psi_{0}\right) \\
& =\left(\Psi_{0},\left(\hat{a}_{1 k}^{\dagger}\right)^{2} \hat{a}_{1 k} \Psi_{0}\right)+2\left(\Psi_{0}, \hat{a}_{1 k}^{\dagger} \Psi_{0}\right) \\
& =2\left(\hat{a}_{1 k} \Psi_{0}, \Psi_{0}\right)=0 .
\end{aligned}
$$

Remark 5.1. We considered the Lagrangian function (3.3) and the Hamiltonian operator (3.10), determining the indefinite constant in (2.3) by (2.18) or in Remark 3.1. On the other hand, in many references (cf. [11], [29] and [32]) the indefinite constant is chosen to be 0 . Consequently, the term $\infty=(1 / 2) \sum_{j=1}^{n} e_{j}^{2} /\left|x^{(j)}-x^{(j)}\right|$ appears in (4.2) from (2.21) and the ground state energy of $H_{\text {rad }}$ is $\sum_{k \in \Lambda^{\prime}} h c|k| / 2$, which tends to infinity as $M_{3}$ tends to infinity. Arguments are made about these infinities in $\S 9-3$ and $\S 9-5$ of [11]. In the present paper we could see that the infinity arising from the term $(1 / 2) \sum_{j=1}^{n} e_{j}^{2} /\left|x^{(j)}-x^{(j)}\right|$ disappears in (4.2) and that the ground state energy of $H_{\text {rad }}$ is 0 .

\section{Preliminaries for the proofs of main results}

From $\S 6$ to $\S 9$ we often write $\vec{x}$ and $\vec{y}$ in $R^{3 n}$ as $x$ and $y$, respectively for the sake of simplicity when no confusion arises.

Let $0 \leq s<t \leq T$. For $x$ and $y$ in $R^{3 n}$ we define

$$
\vec{q}_{x, y}^{t, s}(\theta):=x-\frac{t-\theta}{t-s}(x-y), s \leq \theta \leq t
$$

For $X$ and $Y$ in $R^{4 N}$ we also define

$$
a_{\Lambda^{\prime} X, Y}^{t, s}(\theta):=X-\frac{t-\theta}{t-s}(X-Y), s \leq \theta \leq t .
$$

Then $a_{\Lambda X, Y}^{t, s}(\theta) \in R^{8 N}$ is defined by means of (2.13). We set

$$
V_{1}(x):=\frac{2 \pi}{|V|} \sum_{k \in \Lambda_{1}} \sum_{j, l=1, j \neq l}^{n} \frac{e_{j} e_{l} \cos k \cdot\left(x^{(j)}-x^{(l)}\right)}{|k|^{2}}
$$


and

$$
V_{2}\left(a_{\Lambda^{\prime}}\right):=\sum_{k \in \Lambda^{\prime}, \mathrm{i}, \mathrm{l}}\left(\frac{(c|k|)^{2}}{2|V|}\left(a_{1 k}^{(\mathrm{i})}\right)^{2}-\frac{h c|k|}{2}\right) .
$$

For the sake of simplicity we suppose $\Lambda_{2}^{\prime}=\Lambda_{3}^{\prime}\left(=\Lambda^{\prime}\right)$ from $\S 6$ to $\S 9$. We write $\mathbf{x}=(x, X) \in R^{3 n+4 N}$ and

$$
\mathbf{q}_{\mathbf{x}, \mathbf{y}}^{t, s}(\theta)=\left(\theta, \vec{q}_{x, y}^{t, s}(\theta), a_{\Lambda^{\prime} X, Y}^{t, s}(\theta)\right) \in R^{1+3 n+4 N}, s \leq \theta \leq t
$$

for $s<t$. Then from (3.3) and (3.5) we have

$$
\begin{aligned}
& S_{c}\left(t, s ; \vec{q}_{x, y}^{t, s}, a_{\Lambda X, Y}^{t, s}\right)=\frac{1}{2(t-s)} \sum_{j=1}^{n} m_{j}\left|x^{(j)}-y^{(j)}\right|^{2} \\
& \quad+\int_{\mathbf{q}_{\mathbf{x}, \mathbf{y}}^{t, s}}\left(-V_{1}(x) d t+\frac{1}{c} \sum_{j=1}^{n} e_{j} \tilde{A}\left(x^{(j)}, a_{\Lambda^{\prime}}\right) \cdot d x^{(j)}-V_{2}\left(a_{\Lambda^{\prime}}\right) d t\right)+\frac{|X-Y|^{2}}{2|V|(t-s)} \\
& =\frac{1}{2(t-s)} \sum_{j=1}^{n} m_{j}\left|x^{(j)}-y^{(j)}\right|^{2}-\int_{s}^{t} V_{1}\left(x-\frac{t-\theta}{t-s}(x-y)\right) d \theta \\
& +\frac{1}{c} \sum_{j=1}^{n} e_{j}\left(x^{(j)}-y^{(j)}\right) \cdot \int_{0}^{1} \tilde{A}\left(x^{(j)}-\theta\left(x^{(j)}-y^{(j)}\right), X-\theta(X-Y)\right) d \theta \\
& \quad+\frac{|X-Y|^{2}}{2|V|(t-s)}-\int_{s}^{t} V_{2}\left(X-\frac{t-\theta}{t-s}(X-Y)\right) d \theta \\
& =\frac{1}{2(t-s)} \sum_{j=1}^{n} m_{j}\left|x^{(j)}-y^{(j)}\right|^{2}-(t-s) \int_{0}^{1} V_{1}(x-\theta(x-y)) d \theta \\
& \quad+\frac{1}{c} \sum_{j=1}^{n} e_{j}\left(x^{(j)}-y^{(j)}\right) \cdot \int_{0}^{1} \tilde{A}\left(x^{(j)}-\theta\left(x^{(j)}-y^{(j)}\right), X-\theta(X-Y)\right) d \theta \\
& \quad+\frac{|X-Y|^{2}}{2|V|(t-s)}-(t-s) \int_{0}^{1} V_{2}(X-\theta(X-Y)) d \theta .
\end{aligned}
$$

Let $M \geq 0$ and $p(x, w, X, W)$ a $C^{\infty}$ function in $R^{6 n} \times R^{8 N}$ such that

$$
\left|\partial_{w}^{\alpha} \partial_{x}^{\beta} \partial_{W}^{\alpha^{\prime}} \partial_{X}^{\beta^{\prime}} p(x, w, X, W)\right| \leq C_{\alpha, \beta, \alpha^{\prime}, \beta^{\prime}}(<x ; w><X ; W>)^{M}
$$

for all multi-indices $\alpha, \beta, \alpha^{\prime}$ and $\beta^{\prime}$ with constants $C_{\alpha, \beta, \alpha^{\prime}, \beta^{\prime}}$, where $<x ; w>:=$ 
$\sqrt{1+|x|^{2}+|w|^{2}}$. For $f(x, X) \in \mathcal{S}\left(R^{3 n+4 N}\right)$ we define the operator $P(t, s)$ by

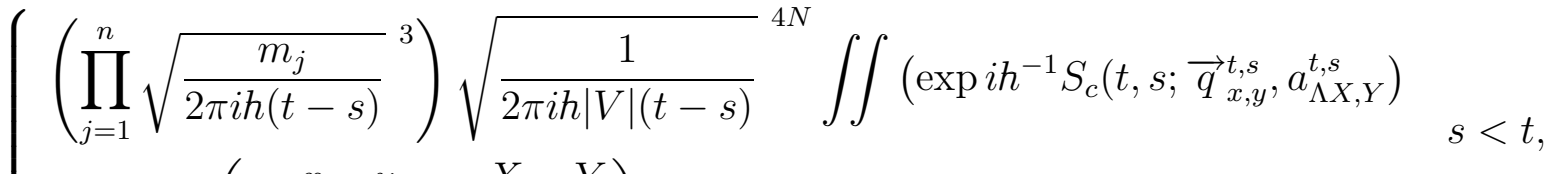

$$
\begin{aligned}
& \times p\left(x, \frac{x-y}{\sqrt{t-s}}, X, \frac{X-Y}{\sqrt{t-s}}\right) f(y, Y) d y d Y, \\
& \left(\prod_{j=1}^{n}{\sqrt{\frac{m_{j}}{2 \pi i h}}}^{3}\right){\sqrt{\frac{1}{2 \pi i \hbar|V|}} \text { Os }-\iint\left\{\operatorname { e x p } i h ^ { - 1 } \left(\sum_{j=1}^{n} \frac{m_{j}\left|w_{j}\right|^{2}}{2}\right.\right.}_{s=t .} \quad \\
& \left.\left.+\frac{|W|^{2}}{2|V|}\right)\right\} p(x, w, X, W) d w d W f(x, X),
\end{aligned}
$$

When $p(x, w, X, W)=1, P(t, s)$ is called the fundamental operator and denoted by $\mathcal{C}(t, s)$.

Lemma 6.1. Let $M_{1}$ and $M_{2}$ be non-negative constants. Suppose that $g(x)\left(x \in R^{3}\right)$ and $\psi(\theta)(\theta \in R)$ in (3.4) satisfy

$$
\left|\partial_{x}^{\alpha} g(x)\right| \leq C_{\alpha}<x>^{M_{1}}, x \in R^{3}
$$

for all $\alpha$ and

$$
\left|\frac{d^{k}}{d \theta^{k}} \psi(\theta)\right| \leq C_{k}<\theta>^{M_{2}}, \theta \in R
$$

for all $k=0,1, \ldots$ Let $f \in \mathcal{S}\left(R^{3 n+4 N}\right)$. Then, $\partial_{x}^{\alpha} \partial_{X}^{\alpha^{\prime}}(P(t, s) f)(x, X)$ are continuous in $0 \leq s \leq t \leq T$ and $(x, X) \in R^{3 n+4 N}$ for all $\alpha$ and $\alpha^{\prime}$.

Proof. Let $s<t$ and make the change of variables: $y \rightarrow w=(x-y) / \sqrt{t-s}$ and $Y \rightarrow W=(X-Y) / \sqrt{t-s}$ in (6.8). Then from (6.6) we have

$$
\begin{aligned}
& P(t, s) f=\left(\prod_{j=1}^{n}{\sqrt{\frac{m_{j}}{2 \pi i \hbar}}}^{3}\right) \sqrt{\frac{1}{2 \pi i \hbar|V|}}^{4 N} \text { Os }-\iint\left(\exp i h^{-1} \phi(t, s ; x, w, X, W)\right) \\
& \times p(x, w, X, W) f(x-\sqrt{\rho} w, X-\sqrt{\rho} W) d w d W, \quad \rho=t-s
\end{aligned}
$$


where

$$
\begin{aligned}
& \phi(t, s ; x, w, X, W):=\sum_{j=1}^{n} \frac{m_{j}}{2}\left|w^{(j)}\right|^{2}+\frac{1}{2|V|}|W|^{2}+\psi(t, s ; x, \sqrt{\rho} w, X, \sqrt{\rho} W) \\
& :=\sum_{j=1}^{n} \frac{m_{j}}{2}\left|w^{(j)}\right|^{2}+\frac{1}{2|V|}|W|^{2}-\rho \int_{0}^{1} V_{1}(x-\theta \sqrt{\rho} w) d \theta+\frac{1}{c} \sum_{j=1}^{n} e_{j} \sqrt{\rho} w^{(j)} \\
& \cdot \int_{0}^{1} \tilde{A}\left(x^{(j)}-\theta \sqrt{\rho} w^{(j)}, X-\theta \sqrt{\rho} W\right) d \theta-\rho \int_{0}^{1} V_{2}(X-\theta \sqrt{\rho} W) d \theta
\end{aligned}
$$

We note from (6.8) that (6.9) is also true for $t=s$.

Let $L^{(j)}:=<w^{(j)}>^{-2}\left(1-i \hbar m_{j}^{-1} \sum_{k=1}^{3} w_{k}^{(j)} \partial / \partial w_{k}^{(j)}\right) \quad(j=1,2, \ldots, n)$ and $L_{1}:=<W>^{-2}\left(1-i \hbar|V| \sum_{k=1}^{4 N} W_{k} \partial_{W_{k}}\right)$. Then, integrating by parts with respect to $w$ and $W$ in (6.9) by means of $L^{(j)}$ and $L_{1}$, we see that the integrand is bounded by

$$
\text { Const. }<x ; X>^{l}<w>^{-(3 n+1)}<W>^{-(4 N+1)}
$$

for some real constant $l$. See the proof of Lemma 2.1 in [19] for further details. Consequently, we see that $(P(t, s) f)(x, X)$ is continuous in $0 \leq s \leq t \leq T$ and $(x, X) \in R^{3 n+4 N}$. We note (6.9) and (6.10). Then, in the same way as in the above we can prove that $\partial_{x}^{\alpha} \partial_{X}^{\alpha^{\prime}}(P(t, s) f)(x, X)$ are continuous in $0 \leq s \leq t \leq T$ and $(x, X) \in R^{3 n+4 N}$ for all $\alpha$ and $\alpha^{\prime}$.

For $0 \leq \sigma_{1}, \sigma_{2} \leq 1$ we set $\sigma:=\left(\sigma_{1}, \sigma_{2}\right)$ and

$$
\begin{aligned}
& \tau(\sigma):=t-\sigma_{1}(t-s) \in R \\
& \zeta^{(j)}(\sigma):=z^{(j)}+\sigma_{1}\left(x^{(j)}-z^{(j)}\right)+\sigma_{1} \sigma_{2}\left(y^{(j)}-x^{(j)}\right) \in R^{3}, j=1,2, \ldots, n \\
& \zeta(\sigma):=z+\sigma_{1}(x-z)+\sigma_{1} \sigma_{2}(y-x) \in R^{3 n} \\
& \tilde{\zeta}(\sigma):=Z+\sigma_{1}(X-Z)+\sigma_{1} \sigma_{2}(Y-X) \in R^{4 N}
\end{aligned}
$$

We also set

$$
B_{m l}\left(x^{(j)}, a_{\Lambda^{\prime}}\right)=\frac{\partial \tilde{A}_{l}}{\partial x_{m}}\left(x^{(j)}, a_{\Lambda^{\prime}}\right)-\frac{\partial \tilde{A}_{m}}{\partial x_{l}}\left(x^{(j)}, a_{\Lambda^{\prime}}\right)
$$

for $l, m=1,2,3$ and $j=1,2, \ldots, n$. Then from (6.6) we have 
Lemma 6.2. We can write for $s<t$

$$
\begin{aligned}
& S_{c}\left(t, s ; \vec{q}_{z, y}^{t, s}, a_{\Lambda Z, Y}^{t, s}\right)-S_{c}\left(t, s ; \vec{q}_{z, x}^{t, s}, a_{\Lambda Z, X}^{t, s}\right) \\
& =\frac{1}{t-s} \sum_{j=1}^{n} m_{j}\left(x^{(j)}-y^{(j)}\right) \cdot\left(z^{(j)}-\frac{x^{(j)}+y^{(j)}}{2}\right) \\
& +(t-s)(x-y) \cdot \int_{0}^{1} \int_{0}^{1} \sigma_{1} \frac{\partial V_{1}}{\partial x}(\zeta(\sigma)) d \sigma_{1} d \sigma_{2} \\
& +\frac{1}{c} \sum_{j=1}^{n} e_{j}\left(x^{(j)}-y^{(j)}\right) \cdot \int_{0}^{1} \tilde{A}\left(x^{(j)}-\theta\left(x^{(j)}-y^{(j)}\right), X-\theta(X-Y)\right) d \theta \\
& +\frac{1}{c} \sum_{j=1}^{n} \sum_{l, m=1}^{3} e_{j}\left(x_{m}^{(j)}-y_{m}^{(j)}\right)\left(x_{l}^{(j)}-z_{l}^{(j)}\right) \int_{0}^{1} \int_{0}^{1} \sigma_{1} B_{m l}\left(\zeta^{(j)}(\sigma), \tilde{\zeta}(\sigma)\right) d \sigma_{1} d \sigma_{2} \\
& +\frac{1}{c} \sum_{j=1}^{n} e_{j}\left(x^{(j)}-y^{(j)}\right) \cdot\left\{(Z-X) \cdot \int_{0}^{1} \int_{0}^{1} \sigma_{1} \frac{\partial \tilde{A}}{\partial a_{\Lambda^{\prime}}}\left(\zeta^{(j)}(\sigma), \tilde{\zeta}(\sigma)\right) d \sigma_{1} d \sigma_{2}\right\} \\
& +(X-Y) \cdot \frac{1}{c} \sum_{j=1}^{n} \sum_{m=1}^{3} e_{j}\left(x_{m}^{(j)}-z_{m}^{(j)}\right) \int_{0}^{1} \int_{0}^{1} \sigma_{1} \frac{\partial \tilde{A}_{m}}{\partial a_{\Lambda^{\prime}}}\left(\zeta^{(j)}(\sigma), \tilde{\zeta}(\sigma)\right) d \sigma_{1} d \sigma_{2} \\
& +\frac{1}{(t-s)|V|}(X-Y) \cdot\left(Z-\frac{X+Y}{2}\right)^{1} \int_{0}^{1} \sigma_{1} \frac{\partial V_{2}}{\partial a_{\Lambda^{\prime}}}(\tilde{\zeta}(\sigma)) d \sigma_{1} d \sigma_{2} . \\
& \left.+(t-s)(X-Y) \cdot \int_{0}^{1} \int_{0}\right)
\end{aligned}
$$

Proof. We use (6.6). From (6.5) and (6.11) we see

$$
\begin{aligned}
& \int_{\boldsymbol{q}_{\boldsymbol{z}, \boldsymbol{y}}^{t, s}}\left(-V_{1}(x)\right) d t-\int_{\boldsymbol{q}_{\boldsymbol{z}, \boldsymbol{x}}}\left(-V_{1}(x)\right) d t=\sum_{j=1}^{n} \sum_{l=1}^{3} \iint_{\boldsymbol{\Delta}} \partial V_{1} / \partial x_{l}^{(j)} d t \wedge d x_{l}^{(j)} \\
& =\sum_{j=1}^{n} \sum_{l=1}^{3} \int_{0}^{1} \int_{0}^{1} \partial V_{1}(\zeta(\sigma)) / \partial x_{l}^{(j)} \operatorname{det} \frac{\partial\left(\tau(\sigma), \zeta_{l}^{(j)}(\sigma)\right)}{\partial\left(\sigma_{1}, \sigma_{2}\right)} d \sigma_{1} d \sigma_{2} \\
& =\sum_{j=1}^{n} \sum_{l=1}^{3}(t-s)\left(x_{l}^{(j)}-y_{l}^{(j)}\right) \int_{0}^{1} \int_{0}^{1} \sigma_{1} \partial V_{1}(\zeta(\sigma)) / \partial x_{l}^{(j)} d \sigma_{1} d \sigma_{2} \\
& =(t-s)(x-y) \cdot \int_{0}^{1} \int_{0}^{1} \sigma_{1} \frac{\partial V_{1}}{\partial x}(\zeta(\sigma)) d \sigma_{1} d \sigma_{2}
\end{aligned}
$$

where $\boldsymbol{\Delta}=\boldsymbol{\Delta}(t, s, x, y, z)$ is the 2-dimensional plane with oriented boundary consisting of $\left(\theta, \vec{q}_{z, y}^{t, s}(\theta)\right),-\left(\theta, \vec{q}_{z, x}^{t, s}(\theta)\right)$ and $\left(\theta, \vec{q}_{y, x}^{s, s}(\theta)\right)(s \leq \theta \leq t)$, and $\sigma$ in 
(6.11) gives the positive orientation of $\Delta$. So the second term on the rhs of (6.13) appears. In the same way the last term appears. It is easy to show that the first and the 7th terms appear.

As in the proof of (6.14) we have

$$
\begin{aligned}
& \int_{\boldsymbol{q}_{\boldsymbol{z}, \boldsymbol{y}}^{t, s}} \tilde{A}\left(x^{(j)}, a_{\Lambda^{\prime}}\right) \cdot d x^{(j)}-\int_{\boldsymbol{q}_{\boldsymbol{z}, \boldsymbol{x}}^{t, s}} \tilde{A}\left(x^{(j)}, a_{\Lambda^{\prime}}\right) \cdot d x^{(j)} \\
& =\int_{\boldsymbol{q}_{\boldsymbol{x}, \boldsymbol{y}}^{s, s}} \tilde{A}\left(x^{(j)}, a_{\Lambda^{\prime}}\right) \cdot d x^{(j)}+\iint_{\Delta} d\left(\tilde{A}\left(x^{(j)}, a_{\Lambda^{\prime}}\right) \cdot d x^{(j)}\right) \\
& =\left(x^{(j)}-y^{(j)}\right) \cdot \int_{0}^{1} \tilde{A}\left(x^{(j)}-\theta\left(x^{(j)}-y^{(j)}\right), X-\theta(X-Y)\right) d \theta \\
& +\sum_{1 \leq m<l \leq 3} \iint_{\Delta} B_{m l} d x_{m}^{(j)} \wedge d x_{l}^{(j)}-\sum_{k \in \Lambda^{\prime}, \mathrm{i}, 1} \sum_{m=1}^{3} \iint_{\Delta}\left(\partial \tilde{A}_{m} / \partial a_{1 k}^{(\mathrm{i})}\right) d x_{m}^{(j)} \wedge d a_{1 k}^{(\mathrm{i})} \\
& =\left(x^{(j)}-y^{(j)}\right) \cdot \int_{0}^{1} \tilde{A}\left(x^{(j)}-\theta\left(x^{(j)}-y^{(j)}\right), X-\theta(X-Y)\right) d \theta \\
& +\sum_{1 \leq m<l \leq 3}\left\{\left(x_{m}^{(j)}-y_{m}^{(j)}\right)\left(x_{l}^{(j)}-z_{l}^{(j)}\right)-\left(x_{l}^{(j)}-y_{l}^{(j)}\right)\left(x_{m}^{(j)}-z_{m}^{(j)}\right)\right\} \\
& \quad \times \int_{0}^{1} \int_{0}^{1} \sigma_{1} B_{m l}\left(\zeta^{(j)}(\sigma), \tilde{\zeta}(\sigma)\right) d \sigma_{1} d \sigma_{2} \\
& -\sum_{m=1}^{3}\left\{\left(x_{m}^{(j)}-y_{m}^{(j)}\right)(X-Z)-(X-Y)\left(x_{m}^{(j)}-z_{m}^{(j)}\right)\right\} \\
& \quad \int_{0}^{1} \int_{0}^{1} \sigma_{1} \frac{\partial \tilde{A}_{m}}{\partial a_{\Lambda^{\prime}}}\left(\zeta^{(j)}(\sigma), \tilde{\zeta}(\sigma)\right) d \sigma_{1} d \sigma_{2} .
\end{aligned}
$$

So we can complete the proof of (6.13) from (6.6).

Let us define $\Phi_{m}^{(j)}\left(t, s ; x^{(j)}, y^{(j)}, z^{(j)}, X, Y, Z\right) \in R(m=1,2,3, j=1,2, \ldots, n)$ 
and $\Phi_{1}(t, s ; x, y, z, X, Y, Z) \in R^{4 N}$ by

$$
\begin{aligned}
& \Phi_{m}^{(j)}=\left(z_{m}^{(j)}-\frac{x_{m}^{(j)}+y_{m}^{(j)}}{2}\right) \\
& +\frac{e_{j}(t-s)}{m_{j} c} \sum_{l=1}^{3}\left(x_{l}^{(j)}-z_{l}^{(j)}\right) \int_{0}^{1} \int_{0}^{1} \sigma_{1} B_{m l}\left(\zeta^{(j)}(\sigma), \tilde{\zeta}(\sigma)\right) d \sigma_{1} d \sigma_{2} \\
& -\frac{e_{j}(t-s)}{m_{j} c}(X-Z) \cdot \int_{0}^{1} \int_{0}^{1} \sigma_{1} \frac{\partial \tilde{A}_{m}}{\partial a_{\Lambda^{\prime}}}\left(\zeta^{(j)}(\sigma), \tilde{\zeta}(\sigma)\right) d \sigma_{1} d \sigma_{2} \\
& +\frac{e_{j}(t-s)}{m_{j} c} \int_{0}^{1} \tilde{A}_{m}\left(x^{(j)}-\theta\left(x^{(j)}-y^{(j)}\right), X-\theta(X-Y)\right) d \theta \\
& +\frac{(t-s)^{2}}{m_{j}} \int_{0}^{1} \int_{0}^{1} \sigma_{1} \partial V_{1}(\zeta(\sigma)) / \partial x_{m}^{(j)} d \sigma_{1} d \sigma_{2}
\end{aligned}
$$

and

$$
\begin{aligned}
& \Phi_{1}=\left(Z-\frac{X+Y}{2}\right)+\frac{(t-s)|V|}{c} \sum_{j=1}^{n} \sum_{m=1}^{3} e_{j}\left(x_{m}^{(j)}-z_{m}^{(j)}\right) \\
& \times \int_{0}^{1} \int_{0}^{1} \sigma_{1} \frac{\partial \tilde{A}_{m}}{\partial a_{\Lambda^{\prime}}}\left(\zeta^{(j)}(\sigma), \tilde{\zeta}(\sigma)\right) d \sigma_{1} d \sigma_{2} \\
&+(t-s)^{2}|V| \int_{0}^{1} \int_{0}^{1} \sigma_{1} \frac{\partial V_{2}}{\partial a_{\Lambda^{\prime}}}(\tilde{\zeta}(\sigma)) d \sigma_{1} d \sigma_{2},
\end{aligned}
$$

respectively. Let $\Phi^{(j)}:=\left(\Phi_{1}^{(j)}, \Phi_{2}^{(j)}, \Phi_{3}^{(j)}\right) \in R^{3}$. Then it follows from (6.13), (6.16) and (6.17) that

$$
\begin{aligned}
& S_{c}\left(t, s ; \vec{q}_{z, y}^{t, s}, a_{\Lambda Z, Y}^{t, s}\right)-S_{c}\left(t, s ; \vec{q}_{z, x}^{t, s}, a_{\Lambda Z, X}^{t, s}\right) \\
& =\frac{1}{t-s} \sum_{j=1}^{n} m_{j}\left(x^{(j)}-y^{(j)}\right) \cdot \Phi^{(j)}\left(t, s ; x^{(j)}, y^{(j)}, z^{(j)}, X, Y, Z\right) \\
& +\frac{1}{(t-s)|V|}(X-Y) \cdot \Phi_{1}(t, s ; x, y, z, X, Y, Z) .
\end{aligned}
$$

\section{The stability of the fundamental operator}

Lemma 7.1. Let $f \in C^{1}\left(R^{d}\right)$ and $\left|\partial_{x}^{\alpha} f\right| \leq C_{\alpha}<x>^{-\left(1+\delta_{\alpha}\right)}$ for all $x \in R^{d}$ and all $|\alpha|=1$, where $\delta_{\alpha}>0$ are constants. Then we have: (1) $f$ is a bounded 
function in $R^{d}$. (2) We have

$$
\begin{aligned}
& |x-z|\left|\partial_{x}^{\alpha} \partial_{y}^{\beta} \partial_{z}^{\gamma} \int_{0}^{1} \int_{0}^{1} \sigma_{1} f\left(z+\sigma_{1}(x-z)+\sigma_{1} \sigma_{2}(y-x)\right) d \sigma_{1} d \sigma_{2}\right| \\
& \leq C_{\alpha, \beta, \gamma}, \quad|\alpha+\beta+\gamma|=1, x, y, z \in R^{d} .
\end{aligned}
$$

The proof is easy, following the proof of Lemma 3.5 in [18].

We note (3.4) and (6.11). Then, it follows from Lemma 7.1 that under the assumptions of Theorem 3.1 we have

$$
\begin{aligned}
& \left|\partial_{x^{(j)}}^{\alpha} \partial_{y^{(j)}}^{\beta} \partial_{z^{(j)}}^{\gamma} \partial_{X}^{\alpha^{\prime}} \partial_{Y}^{\beta^{\prime}} \partial_{Z}^{\gamma^{\prime}}(Z-X) \cdot \int_{0}^{1} \int_{0}^{1} \sigma_{1} \frac{\partial \tilde{A}_{m}}{\partial a_{\Lambda^{\prime}}}\left(\zeta^{(j)}(\sigma), \tilde{\zeta}(\sigma)\right) d \sigma_{1} d \sigma_{2}\right| \\
& \leq C_{\alpha, \beta, \gamma, \alpha^{\prime}, \beta^{\prime}, \gamma^{\prime}}, \quad\left|\alpha+\beta+\gamma+\alpha^{\prime}+\beta^{\prime}+\gamma^{\prime}\right| \geq 0
\end{aligned}
$$

for $x^{(j)}, y^{(j)}, z^{(j)} \in R^{3}$ and $X, Y, Z \in R^{4 N}$. In the same way we have the same estimates as the above for $\left(x_{l}^{(j)}-z_{l}^{(j)}\right) \int_{0}^{1} \int_{0}^{1} \sigma_{1} B_{m l}\left(\zeta^{(j)}(\sigma), \tilde{\zeta}(\sigma)\right) d \sigma_{1} d \sigma_{2}$ and $\left(x_{m}^{(j)}-z_{m}^{(j)}\right) \int_{0}^{1} \int_{0}^{1} \sigma_{1} \frac{\partial \tilde{A}_{m}}{\partial a_{\Lambda^{\prime}}}\left(\zeta^{(j)}(\sigma), \tilde{\zeta}(\sigma)\right) d \sigma_{1} d \sigma_{2}$. To obtain these estimates we assumed (3.6) and (3.7). Consequently, letting $\Theta$ be a component of $\Phi^{(j)}$ and $\Phi_{1}$, and $\left|\alpha+\beta+\gamma+\alpha^{\prime}+\beta^{\prime}+\gamma^{\prime}\right| \geq 1$, then from (6.16) and (6.17) we obtain

$$
\left|\partial_{x}^{\alpha} \partial_{y}^{\beta} \partial_{z}^{\gamma} \partial_{X}^{\alpha^{\prime}} \partial_{Y}^{\beta^{\prime}} \partial_{Z}^{\gamma^{\prime}} \Theta\right| \leq C_{\alpha, \beta, \gamma, \alpha^{\prime}, \beta^{\prime}, \gamma^{\prime}}
$$

together with (6.3) and (6.4) for $0 \leq s \leq t \leq T, x, y, z \in R^{3 n}$ and $X, Y, Z \in$ $R^{4 N}$.

Proposition 7.2. Under the assumptions of Theorem 3.1 we have : (1) There exists a constant $\rho^{*}>0$ such that the mapping $: R^{3 n+4 N} \ni(z, Z) \rightarrow$ $(\xi, \Xi)=\left(\Phi, \Phi_{1}\right):=\left(\Phi^{(1)}, \Phi^{(2)}, \ldots, \Phi^{(n)}, \Phi_{1}\right) \in R^{3 n+4 N}$ is homeomorphic and $\operatorname{det} \partial(\xi, \Xi) / \partial(z, Z) \geq 1 / 2$ for each fixed $0 \leq t-s \leq \rho^{*}, x, y, X$ and $Y$. We write its inverse mapping as $R^{3 n+4 N} \ni(\xi, \Xi) \rightarrow(z, Z)=(z(t, s ; x, \xi, y, X, \Xi, Y)$, $Z(t, s ; x, \xi, y, X, \Xi, Y)) \in R^{3 n+4 N}$. (2) Let $\eta(t, s ; x, \xi, y, X, \Xi, Y)$ be a component of $z$ and $Z$. Then, letting $\left|\alpha+\beta+\gamma+\alpha^{\prime}+\beta^{\prime}+\gamma^{\prime}\right| \geq 1$, we have

$$
\left|\partial_{\xi}^{\alpha} \partial_{x}^{\beta} \partial_{y}^{\gamma} \partial_{\Xi}^{\alpha^{\prime}} \partial_{X}^{\beta^{\prime}} \partial_{Y}^{\gamma^{\prime}} \eta(t, s ; x, \xi, y, X, \Xi, Y)\right| \leq C_{\alpha, \beta, \gamma, \alpha^{\prime}, \beta^{\prime}, \gamma^{\prime}}
$$

for $0 \leq t-s \leq \rho^{*}, x, \xi, y \in R^{3 n}$ and $X, \Xi, Y \in R^{4 N}$. 
Proof. (1) From (6.16) and (6.17) we can write

$$
\partial\left(\Phi, \Phi_{1}\right) / \partial(z, Z)=I+(t-s) d(t, s ; x, y, z, X, Y, Z)
$$

where $I$ is the identity matrix of degree $3 n+4 N$. We can see as in the proof of (7.2) that each component of $d(t, s ; x, y, z, X, Y, Z)$ satisfies (7.2) for all $\alpha, \beta, \gamma, \alpha^{\prime}, \beta^{\prime}$ and $\gamma^{\prime}$. Hence, applying Theorem 1.22 in [31] to the mapping : $(z, Z) \rightarrow\left(\Phi, \Phi_{1}\right)$, we prove $(1)$.

(2) We see

$$
(\xi, \Xi)=\left(\Phi(t, s ; x, y, z, X, Y, Z), \Phi_{1}(t, s ; x, y, z, X, Y, Z)\right)
$$

with $z=z(t, s ; x, \xi, y, X, \Xi, Y)$ and $Z=Z(t, s ; x, \xi, y, X, \Xi, Y)$. So, (7.3) follows from $(7.2)$ and $\operatorname{det} \partial(\xi, \Xi) / \partial(z, Z) \geq 1 / 2$.

Remark 7.1. Let us consider the general case $\Lambda_{2}^{\prime} \subseteq \Lambda_{3}^{\prime}$ in Proposition 7.2. Then from (3.4) and (6.12) we consider $\tilde{A}\left(x, a_{\Lambda_{2}^{\prime}}\right)$ and $B_{m l}\left(x, a_{\Lambda_{2}^{\prime}}\right)$ in (6.16) and (6.17). Let $\Lambda_{1}^{\prime}$ and $\Lambda_{2}^{\prime}$ be fixed. When $\Lambda_{3}^{\prime}=\Lambda_{2}^{\prime}$, we could determine $\rho^{*}>0$ from (7.4) such that we get $\operatorname{det} \partial\left(\Phi, \Phi_{1}\right) / \partial(z, Z) \geq 1 / 2$ for $0 \leq t-s \leq$ $\rho^{*}, x, y, z \in R^{3 n}$ and $X, Y, Z \in R^{4 N_{3}}$. Let $\Lambda_{3}^{\prime} \supseteq \Lambda_{2}^{\prime}$. Then, direct calculations show

$$
\operatorname{det} \partial\left(\Phi, \Phi_{1}\right) / \partial(z, Z) \geq 1 / 2
$$

for $0 \leq t-s \leq \rho^{*}, x, y, z \in R^{3 n}$ and $X, Y, Z \in R^{4 N_{3}}$ from (6.16) and (6.17) since $(t-s)^{2}|V| \partial^{2} V_{2}\left(a_{\Lambda^{\prime}}\right) / \partial\left(a_{1 k}^{(\mathrm{i})}\right)^{2}=(t-s)^{2}(c|k|)^{2}$ are non-negative. Consequently, we can see that when $\Lambda_{1}^{\prime}$ and $\Lambda_{2}^{\prime}$ are fixed, the constant $\rho^{*}>0$ is taken independently of $\Lambda_{3}^{\prime}\left(\supseteq \Lambda_{2}^{\prime}\right)$.

Theorem 7.3. Let $\rho^{*}>0$ be the constant determined in Proposition 7.2. Then under the assumptions of Theorem 3.1 we can find constants $K_{a} \geq 0$ ( $a=$ $0,1,2, \ldots)$ such that

$$
\|\mathcal{C}(t, s) f\|_{B^{a}} \leq \mathrm{e}^{K_{a}(t-s)}\|f\|_{B^{a}}, 0 \leq t-s \leq \rho^{*}
$$

for all $f\left(x, a_{\Lambda^{\prime}}\right) \in B^{a}\left(R^{3 n+4 N}\right)$. 
Proof. The definition (6.8) says

$$
C(s, s)=\text { Identity }
$$

So (7.5) holds for $t=s$.

Let $0<t-s \leq \rho^{*}$. We take $\chi \in C^{\infty}\left(R^{3 n+4 N}\right)$ with compact support such that $\chi(0)=1$. Let $\epsilon>0$ and $f \in \mathcal{S}\left(R^{3 n+4 N}\right)$. Then from (6.8) and (6.18) we can write

$$
\begin{aligned}
& \mathcal{C}(t, s)^{*} \chi(\epsilon \cdot)^{2} \mathcal{C}(t, s) f=\left\{\prod_{j=1}^{n}\left(\frac{m_{j}}{2 \pi h(t-s)}\right)^{3}\right\}\left(\frac{1}{2 \pi h|V|(t-s)}\right)^{4 N} \iint f(y, Y) d y d Y \\
& \times \iint \chi(\epsilon z, \epsilon Z)^{2} \exp \left\{i h^{-1} S_{c}\left(t, s ; \vec{q}_{z, y}^{t, s}, a_{\Lambda Z, Y}^{t, s}\right)-i h^{-1} S_{c}\left(t, s ; \vec{q}_{z, x}^{t, s}, a_{\Lambda Z, X}^{t, s}\right)\right\} d z d Z \\
& =\left\{\prod_{j=1}^{n}\left(\frac{m_{j}}{2 \pi h(t-s)}\right)^{3}\right\}\left(\frac{1}{2 \pi h|V|(t-s)}\right)^{4 N} \iint f(y, Y) d y d Y \iint \chi(\epsilon z, \epsilon Z)^{2} \\
& \times \exp \left(i \sum_{j=1}^{n}\left(x^{(j)}-y^{(j)}\right) \cdot \frac{m_{j} \Phi^{(j)}}{h(t-s)}+i(X-Y) \cdot \frac{\Phi_{1}}{h|V|(t-s)}\right) d z d Z
\end{aligned}
$$

We can make the change of variables $:(z, Z) \rightarrow(\xi, \Xi)=\left(\Phi, \Phi_{1}\right)$ in $(7.7)$ from Proposition 7.2. Then

$$
\begin{aligned}
& \mathcal{C}(t, s)^{*} \chi(\epsilon \cdot)^{2} \mathcal{C}(t, s) f=\left\{\prod_{j=1}^{n}\left(\frac{m_{j}}{2 \pi h(t-s)}\right)^{3}\right\}\left(\frac{1}{2 \pi h|V|(t-s)}\right)^{4 N} \\
& \times \iint f(y, Y) d y d Y \iint \chi(\epsilon z, \epsilon Z)^{2}\left\{\operatorname { e x p } \left(i \sum_{j=1}^{n}\left(x^{(j)}-y^{(j)}\right) \cdot \frac{m_{j} \xi^{(j)}}{h(t-s)}\right.\right. \\
& \left.\left.+i(X-Y) \cdot \frac{\Xi}{h|V|(t-s)}\right)\right\} \operatorname{det} \frac{\partial(z, Z)}{\partial(\xi, \Xi)} d \xi d \Xi .
\end{aligned}
$$

The equation (7.4) and (2) of Proposition 7.2 show

$$
\operatorname{det} \frac{\partial(z, Z)}{\partial(\xi, \Xi)}=1+(t-s) h(t, s ; x, \xi, y, X, \Xi, Y)
$$

where $h(t, s ; x, \xi, y, X, \Xi, Y)$ satisfies (7.3) for all $\alpha, \beta, \gamma, \alpha^{\prime}, \beta^{\prime}$ and $\gamma^{\prime}$. Conse- 
quently from (2) of Proposition 7.2 we have

$$
\begin{aligned}
& \lim _{\epsilon \rightarrow 0} \mathcal{C}(t, s)^{*} \chi(\epsilon \cdot)^{2} \mathcal{C}(t, s) f=\left(\frac{1}{2 \pi}\right)^{3 n+4 N} \lim _{\epsilon \rightarrow 0} \iint f(y, Y) d y d Y \iint \chi(\epsilon z, \epsilon Z)^{2} \\
& \times\{\exp (i(x-y) \cdot \gamma+i(X-Y) \cdot \Gamma)\} \operatorname{det} \frac{\partial(z, Z)}{\partial(\xi, \Xi)} d \gamma d \Gamma \\
& =f(x, X)+(t-s)\left(\frac{1}{2 \pi}\right)^{3 n+4 N} \mathrm{Os}-\iiint \int\{\exp (i(x-y) \cdot \gamma+i(X-Y) \cdot \Gamma)\} \\
& \times h(t, s ; x, \xi, y, X, \Xi, Y) f(y, Y) d y d Y d \gamma d \Gamma,
\end{aligned}
$$

where $\xi^{(j)}=h(t-s) \gamma^{(j)} / m_{j}(j=1,2, \ldots, n)$ and $\Xi=h|V|(t-s) \Gamma$. We note that the second term on the rhs of (7.9) is a pseudo-differential operator. So, applying the Calderón-Vaillancourt theorem ([5]), we obtain

$$
\begin{aligned}
& \lim _{\epsilon \rightarrow 0}\|\chi(\epsilon \cdot) \mathcal{C}(t, s) f\|^{2}=\lim _{\epsilon \rightarrow 0}\left(\mathcal{C}(t, s)^{*} \chi(\epsilon \cdot)^{2} \mathcal{C}(t, s) f, f\right) \\
& =\left(\lim _{\epsilon \rightarrow 0} \mathcal{C}(t, s)^{*} \chi(\epsilon \cdot)^{2} \mathcal{C}(t, s) f, f\right) \leq\left(1+2 K_{0}(t-s)\right)\|f\|^{2} \\
& \leq \mathrm{e}^{2 K_{0}(t-s)}\|f\|^{2}
\end{aligned}
$$

with a constant $K_{0} \geq 0$. Hence we get (7.5) with $a=0$ by Fatou's lemma.

Let $p(x, w, X, W)$ be a $C^{\infty}$ function satisfying (6.7) with an integer $M \geq 0$. Then we obtain

$$
\|P(t, s) f\| \leq \text { Const. }\|f\|_{B^{M}}
$$

as in the proof of (7.5) with $a=0$. See the proof of Proposition 4.3 in [19] for further details.

Let us recall the expression $(6.9)$ of $\mathcal{C}(t, s) f$. Set $\zeta:=(x, X)$ and let $\kappa=\left(\kappa_{1}, \kappa_{2}, \ldots, \kappa_{3 n+4 N}\right)$ be an arbitrary multi-index. Then we can see that $\partial_{\zeta}^{\kappa}(\mathcal{C}(t, s) f)-\mathcal{C}(t, s)\left(\partial_{\zeta}^{\kappa} f\right)$ and $\zeta^{\kappa}(\mathcal{C}(t, s) f)-\mathcal{C}(t, s)\left(\zeta^{\kappa} f\right)$ are written in the form

$$
\begin{aligned}
& (t-s) \sum_{|\gamma| \leq|\kappa|} \tilde{P}_{\gamma}(t, s)\left(\partial_{\zeta}^{\gamma} f\right):=(t-s) \sum_{|\gamma| \leq|\kappa|}\left(\prod_{j=1}^{n}{\sqrt{\frac{m_{j}}{2 \pi i \hbar}}}^{3}\right) \sqrt{\frac{1}{2 \pi i h|V|}}^{4 N} \\
& \times \operatorname{Os}-\iint\left(\exp i h^{-1} \phi(t, s ; x, w, X, W)\right) p_{\gamma}(t, s ; x, \sqrt{\rho} w, X, \sqrt{\rho} W) \\
& \quad \times\left(\partial_{\zeta}^{\gamma} f\right)(x-\sqrt{\rho} w, X-\sqrt{\rho} W) d w d W
\end{aligned}
$$


respectively, where $p_{\gamma}(t, s ; x, w, X, W)$ satisfies (6.7) with $M=|\kappa|-|\gamma|$ for all $\alpha, \beta, \alpha^{\prime}$ and $\beta^{\prime}$. We can prove these results by induction with respect to $|\kappa|$, using $h \partial_{w^{(j)}} \mathrm{e}^{i m_{j} \hbar^{-1}\left|w^{(j)}\right|^{2} / 2}=i m_{j} w^{(j)} \mathrm{e}^{i m_{j} \hbar^{-1}\left|w^{(j)}\right|^{2} / 2}, h \partial_{W} \mathrm{e}^{i \hbar^{-1}|W|^{2} /(2|V|)}=$ $(i W /|V|) \mathrm{e}^{i \hbar^{-1}|W|^{2} /(2|V|)}$ and the integration by parts in (6.9). See the proof of Lemma 3.2 in [21] for further details.

Let $|\kappa|=a(a=0,1,2, \ldots)$. Then we have

$$
\left\|\partial_{\zeta}^{\kappa}(\mathcal{C}(t, s) f)\right\| \leq\left\|\mathcal{C}(t, s)\left(\partial_{\zeta}^{\kappa} f\right)\right\|+(t-s) \sum_{|\gamma| \leq a}\left\|\tilde{P}_{\gamma}(t, s)\left(\partial_{\zeta}^{\gamma} f\right)\right\| .
$$

Applying (7.5) with $a=0$ and (7.10) to the rhs above, we get

$$
\left\|\partial_{\zeta}^{\kappa}(\mathcal{C}(t, s) f)\right\| \leq e^{K_{0}(t-s)}\left\|\partial_{\zeta}^{\kappa} f\right\|+\text { Const. }(t-s) \sum_{|\gamma| \leq a}\left\|\partial_{\zeta}^{\gamma} f\right\|_{B^{a-|\gamma|}} .
$$

We know from Lemma 2.3 with $s=1$ and $a=b$ in [17] that there exist a constant $\mu_{a} \geq 0$ and $\lambda_{a}(\zeta, \eta)$ satisfying

$$
\left|\partial_{\eta}^{\alpha} \partial_{\zeta}^{\beta} \lambda_{a}(\zeta, \eta)\right| \leq C_{\alpha, \beta}<\zeta ; \eta>^{-a}
$$

for all $\alpha$ and $\beta$, and

$$
\Lambda_{a}\left(\zeta, D_{\zeta}\right)=\left(\mu_{a}+<\zeta>^{a}+<D_{\zeta}>^{a}\right)^{-1}
$$

on $\mathcal{S}$, where $\Lambda_{a}\left(\zeta, D_{\zeta}\right)$ is the pseudo-differential operator with symbol $\lambda_{a}(\zeta, \eta)$. So, using Lemma 2.4 in [17] and the Calderón-Vaillancourt theorem, we have

$$
\begin{aligned}
& \left\|\partial_{\zeta}^{\gamma} f\right\|_{B^{a-|\gamma|}} \leq \text { Const. }\left\|\left(\mu_{a-|\gamma|}+<\zeta>^{a-|\gamma|}+<D_{\zeta}>^{a-|\gamma|}\right) \partial_{\zeta}^{\gamma} f\right\| \\
& =\text { Const. } \|\left\{\left(\mu_{a-|\gamma|}+<\zeta>^{a-|\gamma|}+<D_{\zeta}>^{a-|\gamma|}\right) \partial_{\zeta}^{\gamma} \Lambda_{a}\right\}\left(\mu_{a}+<\zeta>^{a}\right. \\
& \left.\quad+<D_{\zeta}>^{a}\right) f \| \leq \text { Const. }\|f\|_{B^{a}} .
\end{aligned}
$$

Hence we get

$$
\left\|\partial_{\zeta}^{\kappa}(\mathcal{C}(t, s) f)\right\| \leq e^{K_{0}(t-s)}\left\|\partial_{\zeta}^{\kappa} f\right\|+\text { Const. }(t-s)\|f\|_{B^{a}} .
$$

In the same way we get

$$
\left\|\zeta^{\kappa}(\mathcal{C}(t, s) f)\right\| \leq e^{K_{0}(t-s)}\left\|\zeta^{\kappa} f\right\|+\text { Const. }(t-s)\|f\|_{B^{a}}
$$


Thus we obtain

$$
\begin{aligned}
\|\mathcal{C}(t, s) f\|_{B^{a}} & \leq e^{K_{0}(t-s)}\|f\|_{B^{a}}+\text { Const. }(t-s)\|f\|_{B^{a}} \\
& \leq e^{K_{a}(t-s)}\|f\|_{B^{a}} .
\end{aligned}
$$

This completes the proof of Theorem 7.3.

Proposition 7.4. Let $0 \leq t-s \leq \rho^{*}$ and $p(x, w, X, W)$ satisfy (6.7) with an integer $M \geq 0$. Then $P(t, s)$ is a continuous operator from $B^{a}(a=$ $0,1,2, \ldots)$ into $B^{a+M}$.

Proof. Let $\zeta=(x, X)$ and $f \in \mathcal{S}\left(R^{3 n+4 N}\right)$. We also use (6.9) as in the proof of Theorem 7.3. Then we have

$$
\partial_{\zeta}^{\kappa} P(t, s) f=\sum_{\gamma \leq \kappa} P_{\gamma}(t, s) \partial_{\zeta}^{\gamma} f
$$

where $\gamma \leq \kappa$ denotes $\gamma_{j} \leq \kappa_{j}$ for all $j$ and $p_{\gamma}(t, s ; x, w, X, W)$ satisfy (6.7) with $M+|\kappa|-|\gamma|$ as $M$. Using $\zeta=(x, X)=(x-\sqrt{\rho} w, X-\sqrt{\rho} W)+\sqrt{\rho}(w, W)$, we also have

$$
\zeta^{\kappa} P(t, s) f=\sum_{\gamma \leq \kappa} Q_{\gamma}(t, s) \zeta^{\gamma} f
$$

where $q_{\gamma}(t, s ; x, w, X, W)$ satisfy (6.7) with $M+|\kappa|-|\gamma|$ as $M$. Hence from (7.10) and (7.14) we see

$$
\begin{aligned}
\|P(t, s) f\|_{B^{a}} & =\|P(t, s) f\|+\sum_{|\kappa|=a}\left(\left\|\zeta^{\kappa} P(t, s) f\right\|+\left\|\partial_{\zeta}^{\kappa} P(t, s) f\right\|\right) \\
& \leq \text { Const. }\|f\|_{B^{a+M}}
\end{aligned}
$$

\section{The consistency of the fundamental opera- tor}

Let $\mathcal{C}(t, s)$ and $H(t)$ be the fundamental operator defined in $\S 6$ and the operator defined by (3.10) with variables $a_{\Lambda^{\prime}}=a_{\Lambda_{2}^{\prime}}=X$, respectively. 
ThEOREM 8.1. Under the assumptions of Theorem 3.1 there exist integers $M \geq 0, M^{\prime} \geq 0, C^{\infty}$ functions $r(t, s ; x, w, X, W)$ and $r^{\prime}(t, s ; x, w, X, W)$ in $0 \leq s \leq t \leq T,(x, w) \in R^{6 n}$ and $(X, W) \in R^{8 N}$ satisfying (6.7) for all $\alpha, \beta, \alpha^{\prime}$ and $\beta^{\prime}$, respectively such that

$$
\left(i h \frac{\partial}{\partial t}-H(t)\right) \mathcal{C}(t, s) f=\sqrt{t-s} R(t, s) f
$$

and

$$
i \hbar \frac{\partial}{\partial s} \mathcal{C}(t, s) f+\mathcal{C}(t, s) H(s) f=\sqrt{t-s} R^{\prime}(t, s) f
$$

for $f \in \mathcal{S}\left(R_{\vec{x}}^{3 n} \times R_{a_{\Lambda^{\prime}}}^{4 N}\right)$, where $R(t, s)$ and $R^{\prime}(t, s)$ are operators defined by (6.8).

Proof. In this proof we write $x$ and $y$ as $\vec{x}$ and $\vec{y}$, respectively. Let $x$ denote variables in $R^{3}$. We may assume $s<t$ from Lemma 6.1. It follows from (3.10), (6.6) and (6.8) that direct calculations show

$$
\begin{aligned}
& \left(i \hbar \frac{\partial}{\partial t}-H(t)\right) \mathcal{C}(t, s) f=-\left(\prod_{j=1}^{n}{\sqrt{\frac{m_{j}}{2 \pi i \hbar(t-s)}}}^{3} \sqrt{\frac{1}{2 \pi i \hbar|V|(t-s)}}^{4 N}\right. \\
& \times \iint\left(\operatorname { e x p } i h ^ { - 1 } S _ { c } ( t , s ; \vec { q } \frac { t , s } { \vec { x } , \vec { y } } , a _ { \Lambda X , Y } ^ { t , s } ) \left\{r_{1}(t, s ; \vec{x}, \vec{y}, X, Y)\right.\right. \\
& \left.\quad+\frac{i \hbar}{2} r_{2}(t, s ; \vec{x}, \vec{y}, X, Y)\right\} f(\vec{y}, Y) d \vec{y} d Y
\end{aligned}
$$

by means of (6.3) and (6.4), where

$$
\begin{aligned}
r_{1}(t, s ; \vec{x}, \vec{y}, X, Y) & =\partial_{t} S_{c}\left(t, s ; \vec{q} \frac{\vec{x}, \vec{y}}{t, s}, a_{\Lambda X, Y}^{t, s}\right)+\sum_{j=1}^{n} \frac{1}{2 m_{j}}\left|\partial_{x^{(j)}} S_{c}-\frac{e_{j}}{c} \tilde{A}\left(x^{(j)}, X\right)\right|^{2} \\
& +V_{1}(\vec{x})+\frac{|V|}{2}\left|\partial_{X} S_{c}\right|^{2}+V_{2}(X)
\end{aligned}
$$

and

$$
\begin{aligned}
r_{2} & =\frac{3 n+4 N}{t-s}-\sum_{j=1}^{n} \frac{1}{m_{j}} \Delta_{x^{(j)}} S_{c} \\
& +\frac{1}{c} \sum_{j=1}^{n} \frac{e_{j}}{m_{j}}\left(\nabla_{x} \cdot \tilde{A}\right)\left(x^{(j)}, X\right)-|V| \Delta_{X} S_{c}, \quad x \in R^{3}
\end{aligned}
$$


(cf. the proof of Proposition 2.3 in [18]).

Set $\rho=t-s$. From (6.6) we can write

$$
\begin{aligned}
& \partial_{x^{(j)}} S_{c}-\frac{e_{j}}{c} \tilde{A}\left(x^{(j)}, X\right)=\frac{m_{j}\left(x^{(j)}-y^{(j)}\right)}{\rho} \\
& +\frac{e_{j}}{c} \int_{0}^{1}\left\{\tilde{A}\left(x^{(j)}-\theta\left(x^{(j)}-y^{(j)}\right), X-\theta(X-Y)\right)-\tilde{A}\left(x^{(j)}, X\right)\right\} d \theta \\
& +\frac{e_{j}}{c} \sum_{l=1}^{3}\left(x_{l}^{(j)}-y_{l}^{(j)}\right) \int_{0}^{1}(1-\theta) \frac{\partial \tilde{A}_{l}}{\partial x}\left(x^{(j)}-\theta\left(x^{(j)}-y^{(j)}\right), X-\theta(X-Y)\right) d \theta \\
& -\rho \int_{0}^{1}(1-\theta) \frac{\partial V_{1}}{\partial x^{(j)}}(\vec{x}-\theta(\vec{x}-\vec{y})) d \theta \\
& =\frac{m_{j}\left(x^{(j)}-y^{(j)}\right)}{\rho}-\frac{e_{j}}{2 c} \sum_{m=1}^{3}\left(x_{m}^{(j)}-y_{m}^{(j)}\right) \frac{\partial \tilde{A}}{\partial x_{m}}\left(x^{(j)}, X\right) \\
& -\frac{e_{j}}{2 c} \sum_{m=1}^{4 N}\left(X_{m}-Y_{m}\right) \frac{\partial \tilde{A}}{\partial X_{m}}\left(x^{(j)}, X\right)+\frac{e_{j}}{2 c} \sum_{l=1}^{3}\left(x_{l}^{(j)}-y_{l}^{(j)}\right) \frac{\partial \tilde{A}_{l}}{\partial x}\left(x^{(j)}, X\right) \\
& +\rho q_{1}\left(t, s ; \vec{x}, \frac{\vec{x}-\vec{y}}{\sqrt{\rho}}, X, \frac{X-Y}{\sqrt{\rho}}\right)
\end{aligned}
$$

and

$$
\begin{aligned}
& \partial_{X} S_{c}=\frac{X-Y}{|V| \rho}-\rho \int_{0}^{1}(1-\theta) \frac{\partial V_{2}}{\partial X}(X-\theta(X-Y)) d \theta \\
& +\frac{1}{c} \sum_{j=1}^{n} \sum_{l=1}^{3} e_{j}\left(x_{l}^{(j)}-y_{l}^{(j)}\right) \int_{0}^{1}(1-\theta) \frac{\partial \tilde{A}_{l}}{\partial X}\left(x^{(j)}-\theta\left(x^{(j)}-y^{(j)}\right), X-\theta(X-Y)\right) d \theta \\
& =\frac{X-Y}{|V| \rho}+\frac{1}{2 c} \sum_{j=1}^{n} \sum_{l=1}^{3} e_{j}\left(x_{l}^{(j)}-y_{l}^{(j)}\right) \frac{\partial \tilde{A}_{l}}{\partial X}\left(x^{(j)}, X\right)+\rho q_{2}\left(t, s ; \vec{x}, \frac{\vec{x}-\vec{y}}{\sqrt{\rho}}, X, \frac{X-Y}{\sqrt{\rho}}\right) .
\end{aligned}
$$

We can easily see

$$
\begin{aligned}
& -\sum_{k, m=1}^{3}\left(x_{k}^{(j)}-y_{k}^{(j)}\right)\left(x_{m}^{(j)}-y_{m}^{(j)}\right) \frac{\partial \tilde{A}_{k}}{\partial x_{m}}\left(x^{(j)}, X\right) \\
& +\sum_{k, l=1}^{3}\left(x_{k}^{(j)}-y_{k}^{(j)}\right)\left(x_{l}^{(j)}-y_{l}^{(j)}\right) \frac{\partial \tilde{A}_{l}}{\partial x_{k}}\left(x^{(j)}, X\right)=0 .
\end{aligned}
$$


The equations (8.6) - (8.8) show

$$
\begin{aligned}
& \sum_{j=1}^{n} \frac{1}{2 m_{j}}\left|\partial_{x^{(j)}} S_{c}-\frac{e_{j}}{c} \tilde{A}\left(x^{(j)}, X\right)\right|^{2}+\frac{|V|}{2}\left|\partial_{X} S_{c}\right|^{2} \\
& =\frac{1}{2 \rho^{2}} \sum_{j=1}^{n} m_{j}\left|x^{(j)}-y^{(j)}\right|^{2}+\frac{|X-Y|^{2}}{2|V| \rho^{2}}+\sqrt{\rho} q_{3}\left(t, s ; \vec{x}, \frac{\vec{x}-\vec{y}}{\sqrt{\rho}}, X, \frac{X-Y}{\sqrt{\rho}}\right)
\end{aligned}
$$

From (6.6) we also have

$$
\begin{gathered}
\partial_{t} S_{c}\left(t, s ; \vec{q} \underset{\vec{x}, \vec{y}}{t, s}, a_{\Lambda X, Y}^{t, s}\right)=-\frac{1}{2 \rho^{2}} \sum_{j=1}^{n} m_{j}\left|x^{(j)}-y^{(j)}\right|^{2}-V_{1}(\vec{x})-\frac{|X-Y|^{2}}{2|V| \rho^{2}} \\
-V_{2}(X)+\sqrt{\rho} q_{4}\left(t, s ; \vec{x}, \frac{\vec{x}-\vec{y}}{\sqrt{\rho}}, X, \frac{X-Y}{\sqrt{\rho}}\right)
\end{gathered}
$$

Hence together with (8.4) we obtain

$$
r_{1}(t, s ; \vec{x}, \vec{y}, X, Y)=\sqrt{\rho} q_{5}\left(t, s ; \vec{x}, \frac{\vec{x}-\vec{y}}{\sqrt{\rho}}, X, \frac{X-Y}{\sqrt{\rho}}\right) .
$$

From (6.6) or (8.6) - (8.7) the same arguments as for (8.11) show

$$
\begin{aligned}
& \sum_{j=1}^{n} \frac{1}{m_{j}} \Delta_{x^{(j)}} S_{c}+|V| \Delta_{X} S_{c}=\frac{3 n+4 N}{\rho}+\frac{2}{c} \sum_{j=1}^{n} \frac{e_{j}}{m_{j}} \int_{0}^{1}(1-\theta) \\
& \times\left(\nabla_{x} \cdot \tilde{A}\right)\left(x^{(j)}-\theta\left(x^{(j)}-y^{(j)}\right), X-\theta(X-Y)\right) d \theta \\
& +\sqrt{\rho} q_{6}\left(t, s ; \vec{x}, \frac{\vec{x}-\vec{y}}{\sqrt{\rho}}, X, \frac{X-Y}{\sqrt{\rho}}\right)=\frac{3 n+4 N}{\rho} \\
& +\frac{1}{c} \sum_{j=1}^{n} \frac{e_{j}}{m_{j}}\left(\nabla_{x} \cdot \tilde{A}\right)\left(x^{(j)}, X\right)+\sqrt{\rho} q_{7}\left(t, s ; \vec{x}, \frac{\vec{x}-\vec{y}}{\sqrt{\rho}}, X, \frac{X-Y}{\sqrt{\rho}}\right) .
\end{aligned}
$$

Hence together with (8.5) we get

$$
r_{2}(t, s ; \vec{x}, \vec{y}, X, Y)=-\sqrt{\rho} q_{7}\left(t, s ; \vec{x}, \frac{\vec{x}-\vec{y}}{\sqrt{\rho}}, X, \frac{X-Y}{\sqrt{\rho}}\right) .
$$

Thus we could complete the proof of (8.1) from (8.3), (8.11) and (8.13). 
Let us consider (8.2). By direct calculations we see that the lhs of (8.2) is equal to

$$
\begin{aligned}
& -\left(\prod_{j=1}^{n}{\sqrt{\frac{m_{j}}{2 \pi i \hbar(t-s)}}}^{n}\right) \sqrt{\frac{1}{2 \pi i \hbar|V|(t-s)}}^{4 N} \\
& \times \iint\left(\operatorname { e x p } i h ^ { - 1 } S _ { c } ( t , s ; \vec { q } \frac { t , s } { x , } , a _ { \Lambda X , Y } ^ { t , s } ) \left\{r_{1}^{\prime}(t, s ; \vec{x}, \vec{y}, X, Y)\right.\right. \\
& \left.\quad+\frac{i h}{2} r_{2}^{\prime}(t, s ; \vec{x}, \vec{y}, X, Y)\right\} f(\vec{y}, Y) d \vec{y} d Y,
\end{aligned}
$$

where

$$
\begin{aligned}
r_{1}^{\prime}(t, s ; \vec{x}, \vec{y}, X, Y) & =\partial_{s} S_{c}\left(t, s ; \vec{q} \frac{t, s}{\vec{x}, \vec{y}}, a_{\Lambda X, Y}^{t, s}\right)-\sum_{j=1}^{n} \frac{1}{2 m_{j}}\left|\partial_{y^{(j)}} S_{c}+\frac{e_{j}}{c} \tilde{A}\left(y^{(j)}, Y\right)\right|^{2} \\
& -V_{1}(\vec{y})-\frac{|V|}{2}\left|\partial_{Y} S_{c}\right|^{2}-V_{2}(Y)
\end{aligned}
$$

and

$$
\begin{aligned}
r_{2}^{\prime} & =-\frac{3 n+4 N}{t-s}+\sum_{j=1}^{n} \frac{1}{m_{j}} \Delta_{y^{(j)}} S_{c} \\
& +\frac{1}{c} \sum_{j=1}^{n} \frac{e_{j}}{m_{j}}\left(\nabla_{x} \cdot \tilde{A}\right)\left(y^{(j)}, Y\right)+|V| \Delta_{Y} S_{c} .
\end{aligned}
$$

Consequently we can prove (8.2) as in the proof of (8.1).

\section{The proofs of the main results}

We first prove Theorem 3.1. Let $\rho^{*}>0$ be the constant determined in Proposition 7.2 and $\chi \in C^{\infty}\left(R^{3 n+4 N}\right)$ with compact support such that $\chi(0)=1$. We consider bounded operators $K_{j}$ and $K_{j}^{\prime}(j=1,2, \ldots, \nu)$ on $B^{a}\left(R^{3 n+4 N}\right)$. 
Then, it holds for $f \in B^{a}\left(R^{3 n+4 N}\right)$ that

$$
\begin{aligned}
& K_{\nu} \chi(\epsilon \cdot) K_{\nu-1} \chi(\epsilon \cdot) \cdots \chi(\epsilon \cdot) K_{1} \chi(\epsilon \cdot) f-K_{\nu}^{\prime} K_{\nu-1}^{\prime} \cdots K_{1}^{\prime} f \\
& =\sum_{j=1}^{\nu} K_{\nu} \chi(\epsilon \cdot) \cdots \chi(\epsilon \cdot) K_{j+1} \chi(\epsilon \cdot)\left(K_{j}-K_{j}^{\prime}\right) K_{j-1}^{\prime} \cdots K_{1}^{\prime} f \\
& \quad+\sum_{j=0}^{\nu-1} K_{\nu} \chi(\epsilon \cdot) \cdots \chi(\epsilon \cdot) K_{j+1}(\chi(\epsilon \cdot)-1) K_{j}^{\prime} \cdots K_{1}^{\prime} f .
\end{aligned}
$$

Noting (6.1) and (6.2), from (3.5) we have

$$
S_{c}\left(T, 0 ; \vec{q}_{\Delta}, a_{\Lambda \Delta}\right)=\sum_{l=1}^{\nu} S_{c}\left(\tau_{l}, \tau_{l-1} ; \vec{q}_{x^{(l)}, x^{(l-1)}}^{\tau_{l}, \tau_{l-1}}, a_{\Lambda X^{(l)}, X^{(l-1)}}^{\tau_{l}, \tau_{l-1}}\right)
$$

where $X^{(l)}=a_{\Lambda^{\prime}}^{(l)}(l=1,2, \ldots, \nu-1)$ and $X^{(\nu)}=a_{\Lambda^{\prime}}$. So, (3.8) is written as

$$
\lim _{\epsilon \rightarrow 0} \mathcal{C}\left(T, \tau_{\nu-1}\right) \chi(\epsilon \cdot) \mathcal{C}\left(\tau_{\nu-1}, \tau_{\nu-2}\right) \chi(\epsilon \cdot) \cdots \mathcal{C}\left(\tau_{2}, \tau_{1}\right) \chi(\epsilon \cdot) \mathcal{C}\left(\tau_{1}, 0\right) \chi(\epsilon \cdot) f
$$

for $f \in B^{a}\left(R^{3 n+4 N}\right)$. Let $f \in B^{a}\left(R^{3 n+4 N}\right)$ and $|\Delta| \leq \rho^{*}$. We can easily see

$$
\sup _{0<\epsilon \leq 1}\|\chi(\epsilon \cdot) f\|_{B^{a}} \leq \text { Const. }\|f\|_{B^{a}}
$$

and

$$
\lim _{\epsilon \rightarrow 0}\|(\chi(\epsilon \cdot)-1) f\|_{B^{a}}=0 .
$$

Consequently, using Theorem 7.3 and (9.1), we can see that (3.8) is well defined in $B^{a}$, which is written as

$$
\mathcal{C}\left(T, \tau_{\nu-1}\right) \mathcal{C}\left(\tau_{\nu-1}, \tau_{\nu-2}\right) \cdots \mathcal{C}\left(\tau_{2}, \tau_{1}\right) \mathcal{C}\left(\tau_{1}, 0\right) f\left(=\mathcal{C}_{\Delta}(T, 0) f\right)
$$

We also see from Remark 3.5 that there exists (3.8) in $\mathcal{S}$ for $f \in \mathcal{S}$.

Let $0 \leq t_{0} \leq t \leq T$. For a subdivision $\Delta$ of $[0, T]$ we can find $j$ and $l$ such that $j \leq l, \tau_{j-1}<t_{0} \leq \tau_{j}$ and $\tau_{l-1}<t \leq \tau_{l}$, where we take $j=1$ for $t_{0}=0$. Then we define

$$
\begin{aligned}
\mathcal{C}_{\Delta}\left(t, t_{0}\right) f:= & \lim _{\epsilon \rightarrow 0} \mathcal{C}\left(t, \tau_{l-1}\right) \chi(\epsilon \cdot) \mathcal{C}\left(\tau_{l-1}, \tau_{l-2}\right) \chi(\epsilon \cdot) \\
& \cdots \chi(\epsilon \cdot) \mathcal{C}\left(\tau_{j+1}, \tau_{j}\right) \chi(\epsilon \cdot) \mathcal{C}\left(\tau_{j}, t_{0}\right) \chi(\epsilon \cdot) f
\end{aligned}
$$


for $f \in B^{a}$ as was stated in Remark 3.3. Let $|\Delta| \leq \rho^{*}$. Then we have

$$
\mathcal{C}_{\Delta}\left(t, t_{0}\right) f=\mathcal{C}\left(t, \tau_{l-1}\right) \mathcal{C}\left(\tau_{l-1}, \tau_{l-2}\right) \cdots \mathcal{C}\left(\tau_{j+1}, \tau_{j}\right) \mathcal{C}\left(\tau_{j}, t_{0}\right) f
$$

as in the proof of (9.2). Consequently, from (7.5) we have

$$
\left\|\mathcal{C}_{\Delta}\left(t, t_{0}\right) f\right\|_{B^{a}} \leq \mathrm{e}^{K_{a}\left(t-t_{0}\right)}\|f\|_{B^{a}} \quad(a=0,1,2, \ldots)
$$

under the assumptions of Theorem 3.1.

Proposition 9.1. Let $|\Delta| \leq \rho^{*}$. Then, under the assumptions of Theorem 3.1 we can find an integer $M \geq 2$ such that

$$
\left\|\mathcal{C}_{\Delta}\left(t, t_{0}\right) f-\mathcal{C}_{\Delta}\left(t^{\prime}, t_{0}^{\prime}\right) f\right\|_{B^{a}} \leq C_{a}\left(\left|t-t^{\prime}\right|+\left|t_{0}-t_{0}^{\prime}\right|\right)\|f\|_{B^{a+M}}
$$

for $0 \leq t_{0} \leq t \leq T, 0 \leq t_{0}^{\prime} \leq t^{\prime} \leq T$ and $a=0,1,2, \ldots$.

Proof. Let $R(t, s)$ and $R^{\prime}(t, s)$ be the operators defined by (8.1) and (8.2), respectively. We determine $M$ in Proposition 9.1 by $\max \left(M, M^{\prime}, 2\right)$ for $M$ and $M^{\prime}$ in Theorem 8.1. We can easily see

$$
i \hbar\left(\mathcal{C}(t, s) f-\mathcal{C}\left(t^{\prime}, s\right) f\right)=\int_{t^{\prime}}^{t}(H(\theta) \mathcal{C}(\theta, s) f+\sqrt{\theta-s} R(\theta, s) f) d \theta
$$

from (8.1) for $s \leq t^{\prime} \leq t \leq T$. Let $\tau_{j}<t \leq \tau_{j+1}$ and $\tau_{k}<t^{\prime} \leq \tau_{k+1}$. So $j \geq k$ holds. Using the equation just after (9.3) and (9.6), we get

$$
\begin{aligned}
& \quad i \hbar\left(\mathcal{C}_{\Delta}\left(t, t_{0}\right) f-\mathcal{C}_{\Delta}\left(t^{\prime}, t_{0}\right) f\right) \\
& =\int_{t^{\prime}}^{t} H(\theta) \mathcal{C}_{\Delta}\left(\theta, t_{0}\right) f d \theta+\int_{\tau_{j}}^{t} \sqrt{\theta-\tau_{j}} R\left(\theta, \tau_{j}\right) d \theta \mathcal{C}_{\Delta}\left(\tau_{j}, t_{0}\right) f \\
& \quad+\sum_{l=1}^{j-k-1} \int_{\tau_{j-l}}^{\tau_{j-l+1}} \sqrt{\theta-\tau_{j-l}} R\left(\theta, \tau_{j-l}\right) d \theta \mathcal{C}_{\Delta}\left(\tau_{j-l}, t_{0}\right) f \\
& \quad+\int_{t^{\prime}}^{\tau_{k+1}} \sqrt{\theta-\tau_{k}} R\left(\theta, \tau_{k}\right) d \theta \mathcal{C}_{\Delta}\left(\tau_{k}, t_{0}\right) f
\end{aligned}
$$

See the proof of Theorem 4.2 in [21] for further details.

As in the proof of (7.14) we see

$$
\|H(t) f\|_{B^{a}} \leq \text { Const. }\|f\|_{B^{a+M}}
$$


from (3.10) because of $M \geq 2$. We also see

$$
\|R(t, s) f\|_{B^{a}} \leq \text { Const. }\|f\|_{B^{a+M}}
$$

from Proposition 7.4 for $0 \leq t-s \leq \rho^{*}$. Consequently, (9.4) and (9.7) show

$$
\begin{aligned}
& h\left\|\mathcal{C}_{\Delta}\left(t, t_{0}\right) f-\mathcal{C}_{\Delta}\left(t^{\prime}, t_{0}\right) f\right\|_{B^{a}} \\
\leq & \text { Const. } e^{K_{a+M} T}\left(1+\sqrt{\rho^{*}}\right)\left|t-t^{\prime}\right|\|f\|_{B^{a+M}}
\end{aligned}
$$

for $0 \leq t_{0} \leq t^{\prime} \leq t \leq T$. The inequality above holds for $0 \leq t_{0} \leq t^{\prime}, t \leq T$. In the same way we get

$$
\begin{aligned}
& h\left\|\mathcal{C}_{\Delta}\left(t, t_{0}\right) f-\mathcal{C}_{\Delta}\left(t, t_{0}^{\prime}\right) f\right\|_{B^{a}} \\
\leq & \text { Const. } e^{K_{a+M} T}\left(1+\sqrt{\rho^{*}}\right)\left|t_{0}-t_{0}^{\prime}\right|\|f\|_{B^{a+M}}
\end{aligned}
$$

for $0 \leq t_{0}, t_{0}^{\prime} \leq t \leq T$. Hence, we can complete the proof of Proposition 9.1 .

Let $M \geq 2$ be the integer determined in Proposition 9.1. We consider a solution $u(t)$, which is $B^{M}$-valued continuous and $L^{2}$-valued continuously differentiable in $\left[t_{0}, T\right]$, to $(3.9)$ with $u\left(t_{0}\right)=0$ for a $t_{0} \in[0, T)$. Then, noting $M \geq 2$, from (3.9) and (3.10) we can easily see

$$
\frac{d}{d t}(u(t), u(t))=2 \Re\left(\frac{d u}{d t}(t), u(t)\right)=-2 h^{-1} \Re i(H(t) u(t), u(t))=0
$$

and so $u(t)=0$ in $\left[t_{0}, T\right]$, where $\Re a$ for a complex number $a$ denotes the real part of $a$. Consequently we can see for a given $f \in B^{a+M}$ that the solution to (3.9) with $u\left(t_{0}\right)=f$ is determined uniquely in the space of all $B^{M}$-valued continuous and $L^{2}$-valued continuously differentiable functions in $\left[t_{0}, T\right]$.

Let $\left\{\Delta_{j}\right\}_{j=1}^{\infty}$ be a family of subdivisions of $[0, T]$ such that $\left|\Delta_{j}\right| \leq \rho^{*}$ and $\lim _{j \rightarrow \infty}\left|\Delta_{j}\right|=0$. Take an arbitrary $f \in B^{a+2 M}(a=0,1,2, \ldots)$. Then we see from (9.4) and (9.5) that $\left\{\mathcal{C}_{\Delta_{j}}\left(t, t_{0}\right) f\right\}_{j=1}^{\infty}$ is uniformly bounded as a family of $B^{a+2 M}$-valued continuous functions and equicontinuous as a family of $B^{a+M_{-}}$ valued functions in $0 \leq t_{0} \leq t \leq T$, respectively. It follows from the Rellich criterion (cf. Theorem XIII. 65 in [28]) that the embedding map from $B^{M}$ into 
$L^{2}$ is compact. So is the embedding map from $B^{a+2 M}$ into $B^{a+M}$ from (7.12), (7.13) and Lemma 2.5 in [17] with $a=b=1$. Consequently, from Ascoli-Arzelà theorem we can find a subsequence $\left\{\Delta_{j_{k}}\right\}_{k=1}^{\infty}$, which may depend on $f$, such that $\mathcal{C}_{\Delta_{j_{k}}}\left(t, t_{0}\right) f$ converges in $B^{a+M}$ uniformly in $0 \leq t_{0} \leq t \leq T$ as $k \rightarrow \infty$. Since $\mathcal{C}_{\Delta_{j}}\left(t_{0}, t_{0}\right) f=f$ follows from Lemma 6.1 , so $(9.7)-(9.9)$ show that $\lim _{k \rightarrow \infty} \mathcal{C}_{\Delta_{j_{k}}}\left(t, t_{0}\right) f=: U\left(t, t_{0}\right) f$, where $U\left(t, t_{0}\right) f$ is $B^{a+M_{\text {-valued }} \text { continuous }}$ and $B^{a}$-valued continuously differentiable function in $0 \leq t_{0} \leq t \leq T$ satisfying (3.9) with $u\left(t_{0}\right)=f$. Hence, it follows from the uniqueness of solutions to (3.9) proved above that $\mathcal{C}_{\Delta}\left(t, t_{0}\right) f$ converges to $U\left(t, t_{0}\right) f$ in $B^{a+M}$ uniformly in $0 \leq t_{0} \leq t \leq T$ as $|\Delta| \rightarrow 0$.

Take an arbitrary $f \in B^{a}$. Let $\Delta$ and $\Delta^{\prime}$ be subdivisions such that $|\Delta| \leq \rho^{*}$ and $\left|\Delta^{\prime}\right| \leq \rho^{*}$. For any $\epsilon>0$ we can take a $g \in B^{a+2 M}$ such that $\|g-f\|_{B^{a}}<\epsilon$. Then from (9.4) we have

$$
\begin{aligned}
& \left\|\mathcal{C}_{\Delta}\left(t, t_{0}\right) f-\mathcal{C}_{\Delta^{\prime}}\left(t, t_{0}\right) f\right\|_{B^{a}} \leq\left\|\mathcal{C}_{\Delta}\left(t, t_{0}\right) g-\mathcal{C}_{\Delta^{\prime}}\left(t, t_{0}\right) g\right\|_{B^{a}} \\
& \quad+\left\|\mathcal{C}_{\Delta}\left(t, t_{0}\right)(f-g)\right\|_{B^{a}}+\left\|\mathcal{C}_{\Delta^{\prime}}\left(t, t_{0}\right)(f-g)\right\|_{B^{a}} \\
& \leq\left\|\mathcal{C}_{\Delta}\left(t, t_{0}\right) g-\mathcal{C}_{\Delta^{\prime}}\left(t, t_{0}\right) g\right\|_{B^{a+M}}+2 \mathrm{e}^{K_{a} T} \epsilon .
\end{aligned}
$$

So,

$$
\varlimsup_{|\Delta|,\left|\Delta^{\prime}\right| \rightarrow 0} \max _{0 \leq t_{0} \leq t \leq T}\left\|\mathcal{C}_{\Delta}\left(t, t_{0}\right) f-\mathcal{C}_{\Delta^{\prime}}\left(t, t_{0}\right) f\right\|_{B^{a}} \leq 2 \mathrm{e}^{K_{a} T} \epsilon .
$$

Hence, we can see that $\mathcal{C}_{\Delta}\left(t, t_{0}\right) f$ converges in $B^{a}$ uniformly in $0 \leq t_{0} \leq t \leq T$ as $|\Delta| \rightarrow 0$. We write this limit as $W\left(t, t_{0}\right) f$.

Let $f \in B^{a}$. Take $f_{j} \in B^{a+M}$ such that $\lim _{j \rightarrow \infty} f_{j}=f$ in $B^{a}$. From (9.7) we have

$$
i \hbar\left(W\left(t, t_{0}\right) f_{j}-f_{j}\right)=\int_{t_{0}}^{t} H(\theta) W\left(\theta, t_{0}\right) f_{j} d \theta
$$

in $B^{a}$. The inequality $\left\|W\left(t, t_{0}\right) f\right\|_{B^{a}} \leq \mathrm{e}^{K_{a}\left(t-t_{0}\right)}\|f\|_{B^{a}}$ holds from (9.4). So, from Lemma 2.5 in [17] with $a=b=1$ we can see

$$
i \hbar\left(W\left(t, t_{0}\right) f-f\right)=\int_{t_{0}}^{t} H(\theta) W\left(\theta, t_{0}\right) f d \theta
$$

in $B^{a-2}$ and that $W\left(t, t_{0}\right) f$ is $B^{a}$-valued continuous and $B^{a-2}$-valued continuously differentiable in $0 \leq t_{0} \leq t \leq T$. Hence $\lim _{|\Delta| \rightarrow 0} \mathcal{C}_{\Delta}\left(t, t_{0}\right) f\left(=W\left(t, t_{0}\right) f\right)$ 
satisfies (3.9) with $u\left(t_{0}\right)=f$. Thus, we could complete the proof of Theorem 3.1 .

We shall consider the proof of Theorem 3.2. Let $\vec{q}_{x, y}^{t, s}(\theta)$ and $\vec{a}_{\Lambda^{\prime} X, Y}^{t, s}(\theta)$ be the paths defined by (6.1) and (6.2) for $s<t$, respectively. For $\vec{\xi}_{k} \in R^{2}(k \in$ $\Lambda_{1}^{\prime}$ ) we define the path by

$$
\phi_{\vec{\xi}_{k}}^{t, s}(\theta):=\vec{\xi}_{k}+\frac{4 \pi \rho_{k}\left(\vec{q}_{x, y}^{t, s}(\theta)\right)}{|k|^{2}} \in R^{2}, s \leq \theta \leq t
$$

as in (3.12). The path $\phi_{\vec{\xi}_{k}}^{t, s}(\theta) \in R^{2}\left(k \in \Lambda_{1}\right)$ is defined by (2.13). So from (2.16) and (2.17) we have

$$
\xi_{-k}^{(1)}=\xi_{k}^{(1)}, \quad \xi_{-k}^{(2)}=-\xi_{k}^{(2)}
$$

For $k \in \Lambda_{1}$ we can easily see

$$
\begin{aligned}
& |k|^{2}\left|\phi_{\vec{\xi}_{k}}^{t, s}(\theta)\right|^{2}-8 \pi \rho_{k}\left(\vec{q}_{x, y}^{t, s}(\theta)\right) \cdot \phi_{\vec{\xi}_{k}}^{t, s}(\theta) \\
& =|k|^{2}\left|\phi_{\vec{\xi}_{k}}^{t, s}-\frac{4 \pi \rho_{k}}{|k|^{2}}\right|^{2}-\frac{16 \pi^{2}}{|k|^{2}}\left|\rho_{k}\right|^{2} \\
& =|k|^{2}\left|\vec{\xi}_{k}\right|^{2}-\frac{16 \pi^{2}}{|k|^{2}}\left|\rho_{k}\left(\vec{q}_{x, y}^{t, s}(\theta)\right)\right|^{2}
\end{aligned}
$$

So, the classical action for $\tilde{L}$ defined by (3.11) is written as

$$
\begin{aligned}
& S\left(t, s ; \vec{q}_{x, y}^{t, s}, a_{\Lambda X, Y}^{t, s},\left\{\phi_{\vec{\xi}_{k}}^{t, s}\right\}_{k \in \Lambda_{1}}\right) \\
& =S_{c}\left(t, s ; \vec{q}_{x, y}^{t, s}, a_{\Lambda X, Y}^{t, s}\right)+\frac{(t-s)}{4 \pi|V|} \sum_{k \in \Lambda_{1}^{\prime}}|k|^{2}\left|\vec{\xi}_{k}\right|^{2}
\end{aligned}
$$

from (2.21) and (3.3).

Let $\chi_{1} \in C^{\infty}\left(R^{2 N_{1}}\right)$ with compact support such that $\chi_{1}(0)=1$. Let $\epsilon>0$ and $\xi:=\left\{\vec{\xi}_{k}\right\}_{k \in \Lambda_{1}^{\prime}} \in R^{2 N_{1}}$. For $f \in \mathcal{S}\left(R^{3 n+4 N}\right)$ we define $G_{\epsilon}(t, s) f(0 \leq s \leq$ 
$t \leq T)$ by

$$
\begin{cases}\left(\prod_{j=1}^{n}{\sqrt{\frac{m_{j}}{2 \pi i \hbar(t-s)}}}^{3} \sqrt{\frac{1}{2 \pi i h|V|(t-s)}}^{4 N}\left(\prod_{k \in \Lambda_{1}^{\prime}} \frac{|k|^{2}(t-s)}{4 i \pi^{2} h|V|}\right)\right. & s<t, \\ \times \int \cdots \int \mathrm{e}^{i \hbar^{-1} S} \chi_{1}(\epsilon \xi) f(y, Y) d y d Y \prod_{k \in \Lambda_{1}^{\prime}} d \vec{\xi}_{k}, & s=t, \\ f, & s=t)\end{cases}
$$

where $S=S\left(t, s ; \vec{q}_{x, y}^{t, s}, a_{\Lambda X, Y}^{t, s},\left\{\phi_{\vec{\xi}_{k}}^{t, s}\right\}_{k \in \Lambda_{1}}\right)$.

Proposition 9.2. Let $f \in B^{a}\left(R^{3 n+4 N}\right)(a=0,1,2, \ldots)$. Then, under the assumptions of Theorem 3.1 we have

$$
\lim _{\epsilon \rightarrow 0} G_{\epsilon}(t, s) f=\mathcal{C}(t, s) f
$$

in $B^{a}$ for $0 \leq t-s \leq \rho^{*}$.

Proof. In the case $t=s$ the equation (9.15) is clear from (7.6). Let $0<t-s \leq$ $\rho^{*}$ and $f \in \mathcal{S}\left(R^{3 n+4 N}\right)$. From (9.13) we have

$$
\begin{aligned}
& G_{\epsilon}(t, s) f=\left(\prod_{j=1}^{n}{\sqrt{\frac{m_{j}}{2 \pi i \hbar(t-s)}}}^{2}\right) \sqrt{\frac{1}{2 \pi i h|V|(t-s)}}^{4 N} \\
& \times \iint\left(\exp i h^{-1} S_{c}\left(t, s ; \vec{q}_{x, y}^{t, s}, a_{\Lambda X, Y}^{t, s}\right)\right) f(y, Y) d y d Y \\
& \times\left(\prod_{k \in \Lambda_{1}^{\prime}} \frac{|k|^{2}(t-s)}{4 i \pi^{2} h|V|}\right) \int \cdots \int\left(\exp \frac{i(t-s)}{4 \pi h|V|} \sum_{k \in \Lambda_{1}^{\prime}}|k|^{2}\left|\vec{\xi}_{k}\right|^{2}\right) \chi_{1}(\epsilon \xi) \prod_{k \in \Lambda_{1}^{\prime}} d \vec{\xi}_{k} .
\end{aligned}
$$

Let $\vec{\eta}_{k}:=\left(\eta_{k}^{(1)}, \eta_{k}^{(2)}\right) \in R^{2}$ and $\eta:=\left\{\vec{\eta}_{k}\right\}_{k \in \Lambda_{1}^{\prime}}$. We know

$$
\int_{-\infty}^{\infty} \mathrm{e}^{i a \theta^{2}} d \theta=\sqrt{\frac{i \pi}{a}}
$$

for a constant $a>0$. So we can write

$$
G_{\epsilon}(t, s) f=P_{\epsilon}(t, s) f
$$


where

$$
\begin{aligned}
p_{\epsilon}(t, s) & =\left(\prod_{k \in \Lambda_{1}^{\prime}} \frac{|k|^{2}}{i \pi}\right) \int \cdots \int\left(\exp i \sum_{k \in \Lambda_{1}^{\prime}}|k|^{2}\left|\vec{\eta}_{k}\right|^{2}\right) \\
& \times \chi(\epsilon \sqrt{4 \pi h|V| /(t-s)} \eta) \prod_{k \in \Lambda_{1}^{\prime}} d \vec{\eta}_{k} .
\end{aligned}
$$

We see that

$$
\lim _{\epsilon \rightarrow 0} p_{\epsilon}(t, s)=1
$$

pointwise. Letting $q_{\epsilon}(t, s)=p_{\epsilon}(t, s)-1$, we have

$$
P_{\epsilon}(t, s) f-\mathcal{C}(t, s) f=Q_{\epsilon}(t, s) f \text {. }
$$

We consider

$$
\begin{aligned}
& \left\|G_{\epsilon}(t, s) f-\mathcal{C}(t, s) f\right\|^{2}=\left\|P_{\epsilon}(t, s) f-\mathcal{C}(t, s) f\right\|^{2} \\
& =\left(\left(P_{\epsilon}(t, s)-\mathcal{C}(t, s)\right)^{\dagger}\left(P_{\epsilon}(t, s)-\mathcal{C}(t, s)\right) f, f\right) \\
& =\left(Q_{\epsilon}(t, s)^{\dagger} Q_{\epsilon}(t, s) f, f\right) .
\end{aligned}
$$

Hence, we obtain (9.15) as in the proof of Theorem 7.3 in the present paper together with Lemma 2.2 in [17]. See the proof of Lemma 4.1 in [20] for further details.

We can write (3.13) as

$$
\lim _{\epsilon \rightarrow 0} G_{\epsilon}\left(T, \tau_{\nu-1}\right) \chi(\epsilon \cdot) G_{\epsilon}\left(\tau_{\nu-1}, \tau_{\nu-2}\right) \chi(\epsilon \cdot) \cdots G_{\epsilon}\left(\tau_{2}, \tau_{1}\right) \chi(\epsilon \cdot) G_{\epsilon}\left(\tau_{1}, 0\right) \chi(\epsilon \cdot) f
$$

in the same way that (3.8) is written in the above of (9.2). Integrating by parts in (9.18), we see that $\sup _{0<\epsilon \leq 1}\left|p_{\epsilon}(t, s)\right|$ is finite. So the same proof as for (7.5) shows

$$
\sup _{0<\epsilon \leq 1}\left\|G_{\epsilon}(t, s) f\right\|_{B^{a}} \leq C_{a}\|f\|_{B^{a}}, a=0,1,2, \ldots
$$

with constants $C_{a}$ from (9.17). Hence, using (9.1), we can prove Theorem 3.2 as in the proof of the convergence of (3.8) to (9.2) together with (9.15). 
Finally, we will prove Theorem 3.3. As in the proof of (6.15) we get

$$
\begin{aligned}
& \left(\int_{\boldsymbol{q}_{\boldsymbol{z}, \boldsymbol{y}}^{t, s}}-\int_{\boldsymbol{q}_{\boldsymbol{z}, \boldsymbol{x}}^{t, s}}\right)\left\{\frac{1}{c} A_{\mathrm{ex}}\left(t, x^{(j)}\right) \cdot d x^{(j)}-\phi_{\mathrm{ex}}\left(t, x^{(j)}\right) d t\right\} \\
& =\frac{1}{c}\left(x^{(j)}-y^{(j)}\right) \cdot \int_{0}^{1} A_{\mathrm{ex}}\left(s, x^{(j)}-\theta\left(x^{(j)}-y^{(j)}\right)\right) d \theta \\
& -(t-s)\left(x^{(j)}-y^{(j)}\right) \cdot \int_{0}^{1} \int_{0}^{1} \sigma_{1} E_{\mathrm{ex}}\left(\tau(\sigma), \zeta^{(j)}(\sigma)\right) d \sigma_{1} d \sigma_{2} \\
& -\frac{1}{c} \sum_{m=1}^{3}\left(x_{m}^{(j)}-y_{m}^{(j)}\right) \sum_{l=1}^{3}\left(z_{l}^{(j)}-x_{l}^{(j)}\right) \int_{0}^{1} \int_{0}^{1} \sigma_{1} B_{m l}^{\prime}\left(\tau(\sigma), \zeta^{(j)}(\sigma)\right) d \sigma_{1} d \sigma_{2}
\end{aligned}
$$

for $s<t$, where $\left(B_{23}^{\prime}(t, x), B_{31}^{\prime}(t, x), B_{12}^{\prime}(t, x)\right)=B_{\text {ex }}(t, x), B_{l m}^{\prime}=-B_{m l}^{\prime}$, and $\tau(\sigma)$ and $\zeta^{(j)}(\sigma)$ were defined by (6.11). See the proof of Proposition 3.3 in [18] for further details. So, we get the equation (6.18) where the sum over $j=1,2, \ldots, n$ of $(9.21)$ multiplied by $m_{j} e_{j} /(t-s)$ is added to. Hence, under the assumptions of Theorem 3.3 we obtain the same assertion as in Theorem 3.1 in the same way that Theorem 3.1 is proved. In the same way of the proof of Theorem 3.2 we also get the same assertion as in Theorem 3.2 under the assumptions of Theorem 3.3. Thus, we could complete the proof of the main results.

Acknowledgement. The author thanks the referee for many useful suggestions.

\section{References}

[1] S. Albeverio, A. Hahn and A. N. Sengupta, Chern-Simons theory, Hida distribution, and state models, Infinite Dimensional Analysis, Quantum Probability and Related Topics 6 (2003), 65-81.

[2] S. Albeverio, R. J. Høegh-Krohn and S. Mazzuchi, Mathematical theory of Feynman Path Integrals (2nd edition), Lecture Notes in Math. 523, Springer-Verlag, Berlin and Heidelberg, 2008. 
[3] A. Arai, Fock Space and Quantum Field (in Japanese), Nihon Hyoron Co., Tokyo, 2000.

[4] F. A. Berezin and M. A. Shubin, The Schrödinger Equation, Kluwer Academic Publishers, Dordrecht, 1983.

[5] A. P. Calderón and R. Vaillancourt, On the boundedness of pseudodifferential operators, J. Math. Soc. Japan 23 (1971), 374-378.

[6] J. M. Cook, The mathematics of second quantization, Trans. Amer. Math. Soc. 74 (1953), 222-245.

[7] P. A. M. Dirac, The Principles of Quantum Mechanics 4th ed., Oxford Univ. Press, London, 1958.

[8] E. Fermi, Quantum theory of radiation, Rev. Modern Phys. 4 (1932), $87-132$.

[9] R. P. Feynman, Space-time approach to nonrelativistic quantum mechanics, Rev. Modern Phys. 20 (1948), 367-387.

[10] R. P. Feynman, Mathematical formulation of the quantum theory of electrodynamic interaction, Phys. Rev. 80 (1950), 440-457.

[11] R. P. Feynman and A. R. Hibbs, Quantum Mechanics and Path Integrals, McGraw-Hill, New York, 1965.

[12] J. Fröhlich, M. Griesemer and I. M. Sigal, Spectral theory for the standard model of non-relativistic QED, Commun. Math. Phys. 283 (2008), 613646.

[13] I. M. Gel'fand and N. Y. Vilenkin, Generalized Functions. Vol. IV, Applications of Harmonic Analysis, Academic Press, New York-London, 1964.

[14] S. J. Gustafson and I. M. Sigal, Mathematical Concepts of Quantum Mechanics, Springer, Berlin, 2003. 
[15] T. Hida, H.-H. Kuo, J. Potthoff and L. Streit, White Noise, Kluwer Academic Publishers, Dordrecht, 1993.

[16] F. Hiroshima, Functional integral representation of a model in quantum electrodynamics, Rev. Math. Phys. 9 (1997), 489-530.

[17] W. Ichinose, A note on the existence and $h$-dependency of the solution of equations in quantum mechanics, Osaka J. Math. 32 (1995), 327-345.

[18] W. Ichinose, On the formulation of the Feynman path integral through broken line paths, Commun. Math. Phys. 189 (1997), 17-33.

[19] W. Ichinose, On convergence of the Feynman path integral formulated through broken line paths, Rev. Math. Phys. 11 (1999), 1001-1025.

[20] W. Ichinose, The phase space Feynman path integral with gauge invariance and its convergence, Rev. Math. Phys. 12 (2000), 1451-1463.

[21] W. Ichinose, Convergence of the Feynman path integral in the weighted Sobolev spaces and the representation of correlation functions, J. Math. Soc. Japan 55 (2003), 957-983.

[22] W. Ichinose, The continuity of the solution with respect to an electromagnetic potential to the Schrödinger equation and the Dirac equation (preprint).

[23] G. W. Johnson and M. L. Lapidus, The Feynman Integral and Feynman's Operational Calculus, Oxford Univ. Press, Oxford, 2000.

[24] H. Kumano-go, Pseudo-Differential Operators, MIT Press, Cambridge, 1981.

[25] E. H. Lieb and M. Loss, Analysis, AMS, Providence, 1997.

[26] S. Mizohata, The Theory of Partial Differential Equations, Cambridge Univ. Press, New York, 1973. 
[27] E. Nelson, Schödinger particles interacting with a quantized scalar field in Proceedings of a Conference on the Theory and Applications of Analysis in Function Space, M.I.T. Press, Cambridge, 1964, pp. 88-120.

[28] M. Reed and B. Simon, Methods of Modern Mathematical Physics IV: Analysis of Operators, Academic Press, New York, 1978.

[29] J. J. Sakurai, Advanced Quantum Mechanics, Addison-Wesley, Massachusetts, 1967.

[30] F. E. Schroeck, Jr., Generalization of the Cook formalism for Fock space, J. Math. Phys. 12 (1971), 1849-1857.

[31] J. T. Schwartz, Nonlinear Functional Analysis, Gordon and Breach Science Publishers, New York, 1969.

[32] H. Spohn, Dynamics of Charged Particles and Their Radiation Field, Cambridge University Press, Cambridge, 2004.

[33] M. S. Swanson, Path Integrals and Quantum Processes, Academic Press, San Diego, 1992. 